\title{
Aphids of the tribe Macrosiphini (Insecta: Homoptera: Aphididae) in Belarus
}

\section{Тли трибы Macrosiphini (Insecta: Homoptera: Aphididae) Белоруссии}

\author{
S.V. Buga \& A.V. Stekolshchikov \\ C.В. БУГА, А.В. СТЕКОЛЬщИКОВ
}

S.V. Buga, Department of Zoology, Belarusian State University, Nezavisimosti av. 4, 220030 Minsk, Republic of Belarus. E-mail: sergey.buga@gmail.com

A.V. Stekolshchikov, Zoological Institute, Russian Academy of Sciences, Universitetskaya nab. 1, 199034 St Petersburg, Russia.E-mail:aphids@zin.ru

Investigation of Macrosiphini in Belarus started in the 1900s. However, a regional checklist of Macrosiphini has not been published yet. Based on material preserved in our collections and data scattered across publications, we have compiled the first comprehensive list of Macrosiphini recorded from Belarus and briefly analysed it from the geographical and ecological points of view. The list includes 52 genera, 156 species and 2 subspecies. Acyrthosiphon cyparissiae (Koch, 1855), A. euphorbiae Börner, 1949, Diuraphis (Holcaphis) frequens (Walker, 1848), Dysaphis angelicae (Koch, 1854), D. apiifolia petroselini (Börner, 1950), D. lauberti (Börner, 1940), Paramyzus heraclei Börner, 1933, Macrosiphum melampyri Mordvilko, 1919, and Uroleucon pulicariae (Hille Ris Lambers, 1939) are recorded from Belarus for the first time. The recorded diversity is lower than in the adjacent countries - Poland and Latvia. In accordance with the global pattern of aphid relations with their host plants, the majority of Belarusian Macrosiphini species permanently inhabit herbaceous plants (monoecious species) or migrate to them from woody plants (dioecious species). Among the recorded species, 27 inhabit a single host plant species, 85 - plants of a single genus, 37 - plants of a single family, and only seven species inhabit host plants of several families or more. At least 16 species ( $10 \%$ of local fauna of Macrosiphini) are alien to Belarus.

Исследования фауны тлей трибы Macrosiphini в Белоруссии были начаты еще в первые десятилетия XX-го века, однако до настоящего времени ни одного регионального списка видов Macrosiphini не было опубликовано. Целью данной работы было объединение и критическая оценка сведений о Macrosiphini Белоруссии имеющихся в разрозненных публикациях, которые были дополнены данными полученными на основании изучения коллекционных материалов, Это позволило впервые получить аннотированный список видов трибы Macrosiphini обитающих в Белоруссии и провести краткий анализ географических и экологических особенностей фауны Macrosiphini Белоруссии. Список включает 52 родов, 156 видов и 2 подвида. Впервые для Белоруссии отмечены Acyrthosiphon cyparissiae (Koch, 1855), A. euphorbiae Börner, 1949, Diuraphis (Holcaphis) frequens (Walker, 1848), Dysaphis angelicae (Koch, 1854), D. apiifolia petroselini (Börner, 1950), D. lauberti (Börner, 1940), Paramyzus heraclei Börner, 1933, Macrosiphum melampyri Mordvilko, 1919, Uroleucon pulicariae (Hille Ris Lambers, 1939). Выявленное число видов трибы на территории Белоруссии оказалось меньше, чем в соседних странах - Польше и Латвии. Большинство видов Macrosiphini, обитающих в Белоруссии, постоянно живет на травянистых растениях (однодомные виды) или мигрирует на них с древесных растений (двудомные виды). Среди отмеченных видов 27 питается на одном виде растения-хозяина, 85 - на хозяевах из одного рода, 37 - на хозяевах из одного семейства и только семь видов используют растений-хозяев из нескольких семейств. По крайней мере, 16 видов (10\% местной фауны Macrosiphini) являются чужеродными для фауны Белоруссии.

Key words: aphids, fauna, Belarus, Homoptera, Aphididae, Macrosiphini

Ключевые слова: тли, фауна, Белоруссия, Homoptera, Aphididae, Macrosiphini 


\section{INTRODUCTION}

Belarus, a small (207 $\left.600 \mathrm{~km}^{2}\right)$ landlocked Central European country (Fig. 1), is situated in the transitional zone between the European deciduous forests and the North Eurasian evergreen coniferous forests. The country is divided (Fig. 2) into five geographical provinces (Martsinkevich \& Pirozhnik, 2007).

Study of Belarusian aphids started in the late 1900s, when A.K. Mordvilko collected aphids in the central and southern parts of Eastern Belarus. He listed 28 aphid species, including two species of Macrosiphini (Mordvilko, 1930). At the same time several cecidological papers reported cecidia-forming aphids in Western Belarus (territory of Poland at that time). Four species of Macrosiphini were recorded by Z. Fiedorowiczówna (1930), and two species, by J. Perepeczko-Baumanowa (1934).
After the 1940s, aphidological researches were focused on the field of applied entomology. The following species of Macrosiphini were noted as pests of cultivated plants: $A n u-$ raphis subterranea (Walker, 1852), Dysaphis devecta (Walker, 1849), Myzus cerasi (Fabricius, 1775), Phorodon humuli (Schrank, 1801) in orchards (Bezdenko, 1958); $\mathrm{Ma}$ crosiphum rosae (Linnaeus, 1758) damaging roses in ornamental plantings (Losinskaya, 1960); Brachycaudus helichrysi (Kaltenbach, 1843), B. spiraeae Börner, 1932, Cryptomyzus ribis (Linnaeus, 1758), Dysaphis crataegi (Kaltenbach, 1843), D. (Pomaphis) sorbi (Kaltenbach, 1843), Liosomaphis berberidis (Kaltenbach, 1843), Rhopalomyzus (Judenkoa) lonicerae (Siebold, 1839) as pests of ornamental trees and shrubs (Gorlenko \& Pan'ko, 1967, 1972; Pan'ko, 1969); Metopolophium dirhodum (Walker, 1849), Sitobion avenae (Fabricius, 1775) as pests of cereals (Mormyljova, 1981); Myzus (Nectaro-

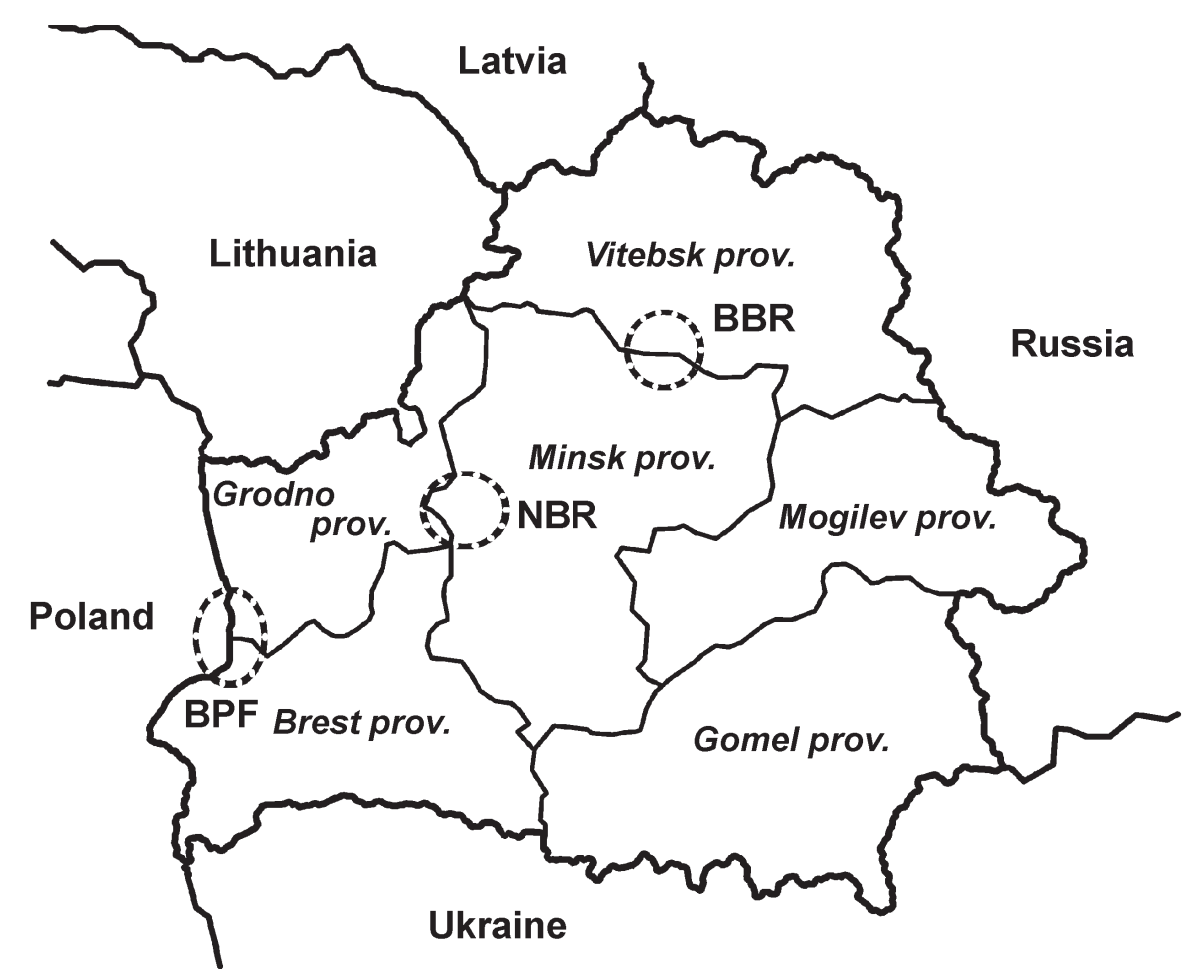

Fig. 1. Administrative provinces of Belarus and main areas of aphid sampling and registration during XX century (BBR-Berezina Biosphere Reserve, BPF-Belavezha Primeval Forest, NBR-Naliboki Biological Reserve). 


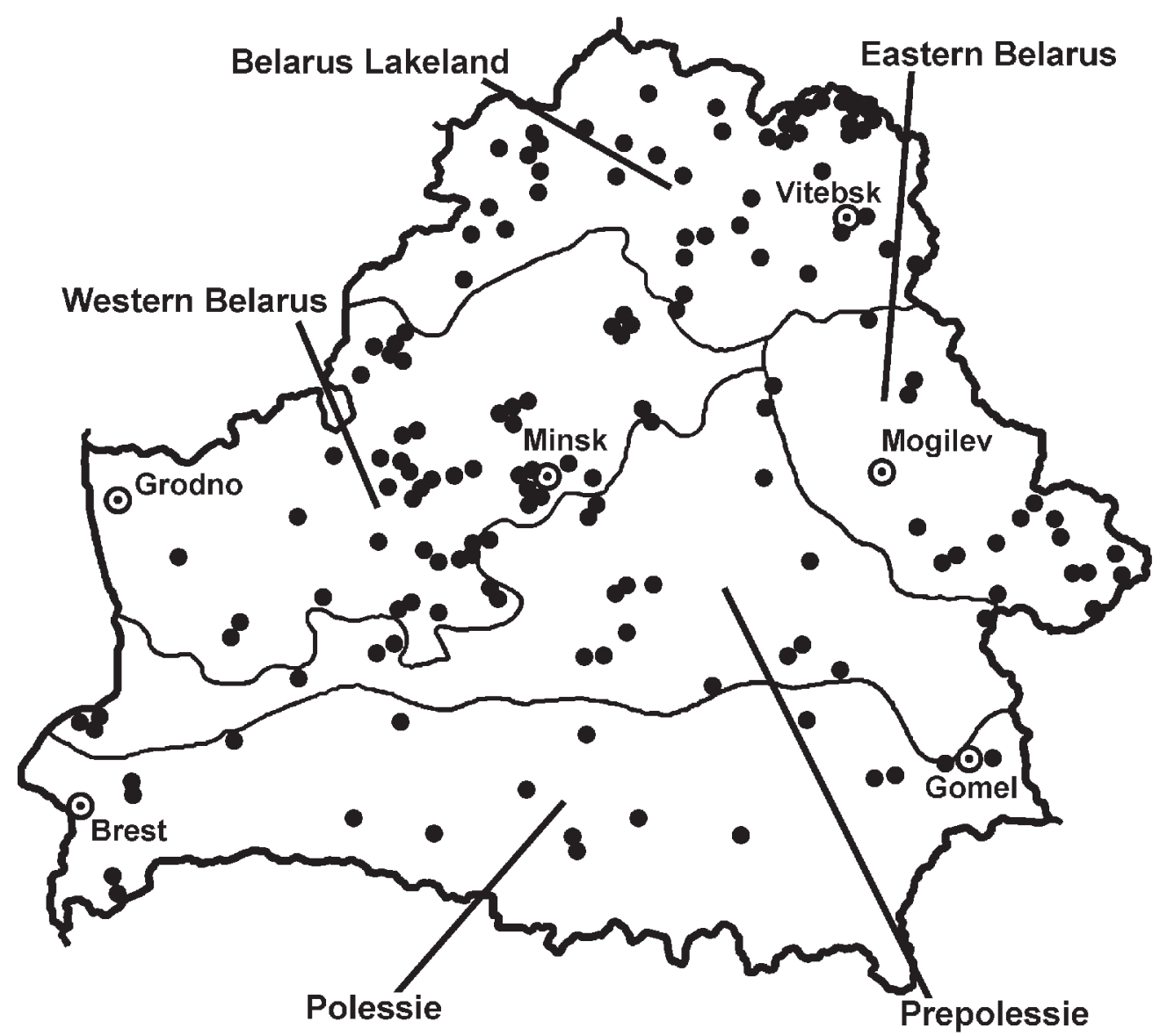

Fig. 2. Landscape provinces of Belarus and geographic points of aphid sampling and registration.

siphon) persicae (Šulzer, 1776), Aulacorthum solani (Kaltenbach, 1843), Macrosiphum euphorbiae (Thomas, 1878) as vectors of potato viruses (Zhukova, 1986).

Systematic study of the aphid fauna started in Belarus in the 1980s. Till now several papers on the local faunas of Belarusian regions have been published (Buga, 1989, 1995; Buga et al., 2008; Rakauskas \& Buga, 2010; Stekolshchikov \& Buga, 2006; Stekolshchikov et al., 2008, 2010); special attention was paid to species damaging woody plants (Buga, 1997, 2001; Buga \& Gorlenka, 1987; Buga \& Stekolshchikov, 2009). However, a general regional checklist of Macrosiphini has not been published yet.

In the present paper we provide a critical evaluation of integrated information on
Macrosiphini of Belarus, based on scattered notes in numerous publications (most of which appeared in obscure regional journals hardly available to foreign scientists) and collections.

\section{MATERIAL AND METHODS}

The paper is based on the material collected by S.V. Buga since 1983. Although this information had been partly published in Russian or Belarusian, we prefer to repeat the collection data in English in the list below. We also list the material collected by Yu.V. Safonova, V.E. Jarigo, I.P. Moskvina, A.A. Mityanin, A.A. Ponomarev, D.L. Petrov, N.V. Leshchinskaya, R. Rakauskas, L.A. Serbina, N.V. Voronova, 
O.L. Nesterova, as well as material preserved in the collection of the Zoological Institute (St Petersburg, Russia).

Microscope slides were prepared using Faure-Berlese mounting fluid or Canada balsam (Blackman \& Eastop, 2000). The material is deposited at the Department of Zoology of Belarusian State University (Minsk, Belarus), at the Zoological Institute (St Petersburg, Russia) and at the Department of Zoology of Vilnius University (Vilnius, Lithuania). Data extracted from publications are given in a uniform manner but the completeness of the data depends on the original publications. When different variants of transliteration of geographical names are more or less widely used in the literature we provide the additional varians in parentheses after the main variant. Aphid synonymy follows Remaudière and Remaudière (1997) with subsequent additions (Blackman \& Eastop, 2011; Eastop \& Blackman, 2005; Holman, 2009). Host plants were identified following Parfenov (1999).

Abbreviations. Aphid's morphs: al. alate viviparous female, apt. - apterous viviparous female, em. - emigrant, fund. fundatrix, gyn. - gynopara, ovip. fem. oviparous female. Localities: BBR - Berezina Biosphere Reserve, BPF - Belovezha Primeval Forest, CBG - Central Botanical Gardens of the National Academy of Science of Belarus, NBR - Naliboki Biological Reserve (Fig. 1), RWS - railway station.

\section{LIST OF SPECIES}

Acaudinum centaureae (Koch, 1854)

Specimens examined. Minsk, CBG, 25 July 2006, Centaurea sp., on leaves, apt., N.V. Leshchinskaya leg.

\section{Acyrthosiphon boreale}

Hille Ris Lambers, 1952

Specimens examined. Minsk Prov, Molodechno Distr., near Udranka Vill., dry meadow, 22 May 2008, Potentilla argentea
L., on lower side of leaves, apt., N.V. Leshchinskaya leg.

\section{Acyrthosiphon caraganae}

(Cholodkovsky, 1908)

Specimens examined. Brest Prov., Kamenets Distr: BPF, Kamenyuki Vill., green stands, 14 Oct. 1999, Caragana arborescens Lam., on lower side of leaves, ovip. fem., S.V. Buga leg.; near Kamenyuki Vill., hamlet Dokudovo, green stands, 14 Oct. 1999 , Caragana arborescens Lam., on lower side of leaves, ovip. fem., S.V. Buga leg. Minsk: CBG, arboretum: 16 Aug. 1985, Caragana arborescens Lam., on lower side of leaves, al., S.V. Buga leg.; 11 July 1986, Caragana arborescens Lam., on green beans, apt., al., S.V. Buga leg. Minsk Prov.: Kopyl' Distr., Sloboda-Kuchinka Vill., green stands, 10 July 2010, Caragana arborescens Lam., on lower side of leaves, apt., S.V. Buga leg.; Minsk Distr.: near Kryzhovka Vill., green stands, 16 June 2001, Caragana arborescens Lam., on leaves, apt., al., S.V. Buga leg.; Zhdanovichi Vill., green stands, 30 June 2001, Caragana arborescens Lam., on maturing beans, apt., S.V. Buga leg.; Nesvizh Distr., 0.2 km N Lipa RWS, birch forest, 6 Sept. 2000, Caragana arborescens Lam., on lower side of leaves, gyn., S.V. Buga leg.; Stolbtsy Distr., Stolbtsy, green stands, 7 July 2000, Caragana arborescens Lam., on lower side of leaves, apt., S.V. Buga leg.; Volozhin Distr., Sakovshchina Vill., green stands, 18 June 1999, Caragana arborescens Lam., on green beans, apt., al., S.V. Buga leg. Vitebsk Prov.: Liozno Distr., Liozno, green stands, 20 June 2008, Caragana arborescens Lam., on maturing beans, apt., S.V. Buga leg.; Polotsk Distr., Novopolotsk, green stands, 2 July 2007, Caragana arborescens Lam., on maturing beans, apt., al., S.V. Buga leg.; Polotsk, green stands, 2 July 2007, Caragana arborescens Lam., on maturing beans, apt., S.V. Buga leg.

Acyrthosiphon cyparissiae (Koch, 1855)

Specimens examined. Gomel Prov:: Mozyr' Distr., Mozyr', 5 July 1929, Euphorbia 
cyparissias L., apt., al., A.K. Mordvilko leg.; Svetlogorsk Distr., Yakimova Sloboda Vill., 22 July 1929, Euphorbia cyparissias L., apt., A.K. Mordvilko leg.

\section{Acyrthosiphon euphorbiae Börner, 1949}

Specimens examined. Gomel Prov. Petrikov Distr., Petrikov, 11 Aug. 1929, Euphorbia ?peplus L., on lower side of leaves, apt., A.K. Mordvilko leg.

Acyrthosiphon lactucae (Passerini, 1860)

Published information. Minsk Prov. (Mordvilko, 1932)

\section{Acyrthosiphon knechteli (Börner, 1950)}

Specimens examined. Minsk Prov., Minsk Distr:: near Kryzhovka Vill., pine forest, 10 July 2000, Vaccinium uliginosum L., on apical part of stem and lower side of leaves, apt., S.V. Buga leg.; same locality, but 16 July 2000, Vaccinium uliginosum L., on apical part of stem and lower side of leaves, al., S.V. Buga leg.

\section{Acyrthosiphon malvae potha \\ Börner, (1943) 1950}

Specimens examined. Minsk Prov. Minsk Distr., near Minsk, dry meadow, 16 May 2007, Potentilla argentea L., on lower side of leaves, apt., N.V. Leshchinskaya leg.

\section{Acyrthosiphon pisum (Harris, 1776)}

Specimens examined. Gomel Prov., Dobrush Distr., $1 \mathrm{~km}$ NE of Dobrush RWS, pine forest, 28 Sept. 2007, Chamaecytisus ruthenicus (Fisch. ex Wol.) Klásková, on lower side of leaves, apt., S.V. Buga leg. Grodno (Grodna) Prov., Smorgon' Distr., 2 km SW Rachuny Vill., pine forest, 4 Oct. 2005, Trifolium alpestre L., on lower side of leaves, ovip. fem., S.V. Buga leg. Mogilev Prov.: Belynichi Distr: 1 km ESE Korytnitsa Vill., clover field, 18 Aug. 2005, Trifolium hybridum L., on lower side of leaves, apt., S.V.
Buga leg.; same locality, but dry meadow, 23 Aug. 2005, Vicia cracca L., on apical part of stem, apt., S.V. Buga leg.; Cherikov Distr., $6 \mathrm{~km}$ WSW Cherikov, pine forest, 24 Aug. 2005, Astragalus arenarius L., on lower side of leaves, apt., S.V. Buga leg.; Khotimsk Distr., $1.5 \mathrm{~km}$ NW Markovka Vill., dry meadow, 24 Aug. 2005, Vicia cracca L., on lower side of leaves, apt., S.V. Buga leg.; Kostyukovichi Distr., 1 km E Krutoy Rov Vill., dry meadow, 25 Aug. 2005, Vicia cracca L., on lower side of leaves, apt., S.V. Buga leg.

\section{Acyrthosiphon (Liporrhinus) chelidonii}

(Kaltenbach, 1843)

Specimens examined. Minsk, ruderal vegetation, 14 July 1996, Chelidonium majus L., on lower side of leaves, apt., Yu.V. Safonova leg.

Amphorophora ampullata Buckton, 1876

Specimens examined. Gomel Prov., Rechitsa Distr., near Kopan' RWS, oak-dominated forest with species-rich grass cover, 31 July 2009, Dryopteris filis-mas (L.) Schott., on fronds, apt., S.V. Buga leg.

\section{Amphorophora idaei (Börner, 1939)}

Specimens examined. Brest Prov.: $\mathrm{Ka}$ menets Distr., BPF, near Kamenyuki Vill., edge of mixed forest, 13 Oct. 1999, Rubus idaeus L., on lower side of leaves, ovip. fem., S.V. Buga leg.; Malorita Distr., vicinity of the Mel'niki Vill., pine forest, 29 Aug. 2009, $R u$ bus idaeus L., on lower side of leaves, apt., S.V. Buga leg. Gomel Prov., Oktyabr'skiy Distr., near Moshny RWS, pine forest, 28 Sept. 2009, Rubus idaeus L., on lower side of leaves, ovip. fem., S.V. Buga leg. Grodno (Grodna) Prov., Iv'e (Iv'ye, Iv'evsk) Distr: $0.3 \mathrm{~km}$ S Yakhimovshchina Vill., spruce-dominated forest, 27 Aug. 2010, Rubus idaeus L., on apical part of stem, S.V. Buga leg.; $0.6 \mathrm{~km}$ S Yakhimovshchina Vill., spruce-dominated forest, 27 Aug. 2010, Rubus idaeus L., on lower side of leaves, apt., S.V. Buga leg. 
Minsk Prov.: Minsk Distr.: Priluki Vill., orchard, 22 Aug. 1985, Rubus idaeus L. cv., on lower side of leaves, apt., S.V. Buga leg.; near Priluki Vill., mixed forest, 27 Aug. 1985, $R u$ bus idaeus L., on lower side of leaves, apt., S.V. Buga leg.; Starye Dorogi Distr., 3 km W Falichi RWS, forest glade, 3 Aug. 2000, $R u$ bus idaeus L., on lower side of leaves, apt., S.V. Buga leg.; Volozhin Distr., NBR, nearby hamlet Pechishche, floodplain alder forest, 25 July 1987, Rubus idaeus L., on lower side of leaves, apt., S.V. Buga leg. Vitebsk Prov., Lepel' Distr.: BBR, near hamlet Savskiy Bor, mixed forest, 15 Aug. 1986, Rubus idaeus L., on lower side of leaves, al., S.V. Buga leg.; 2 km SSE Zaboen'e Vill., spruce-dominated forest, 19 July 2004, Rubus idaeus L., on lower side of leaves, apt., S.V. Buga leg.

\section{Amphorophora rubi (Kaltenbach, 1843)}

Specimens examined. Gomel Prov., Svetlogorsk Distr., Yakimova Sloboda Vill., 26 July 1929, Rubus sp., on apical part of stem, apt., A.K. Mordvilko leg. Minsk, CBG, landscape park, 25 June 1987, Rubus caesius L., on apical part of stem, al., S.V. Buga leg. Minsk Prov.: Borisov Distr., near Leshchiny Vill., spruce-dominated forest, 24 June 1987, Rubus caesius L., on apical part of stem, al., S.V. Buga leg.; Starye Dorogi Distr., $3 \mathrm{~km} \mathrm{~W}$ Falichi RWS, wasteland, 3 Aug. 2000, Rubus caesius L., on lower side of leaves, apt., S.V. Buga leg.

Anuraphis catonii Hille Ris Lambers, 1935

Specimens examined. Minsk Prov.: Molodechno Distr., near Radoshkovichi RWS, dry meadow, 30 May 2008, Pimpinella saxifraga $\mathrm{L}$., on basal part of stem, em., N.V. Leshchinskaya leg.; Stolbtsy Distr., near Belomosh'e Vill., dry meadow, 13 July 2010, Pimpinella saxifraga L., on root colar, apt., S.V. Buga leg.

\section{Anuraphis farfarae (Koch, 1854)}

Specimens examined. Grodno (Grodna) Prov., Iv'e (Iv'ye, Iv'ersk) Distr., near Zubo- vichi Vill., dry meadow, 27 Aug. 2010, Tussilago farfara L., root collar, apt., S.V. Buga leg. Minsk Prov., Minsk Distr., Priluki Vill., fruit orchard, 20 July 1986, Tussilago farfara L., on root collar, apt., al., S.V. Buga leg. Vitebsk Prov.: Lepel' Distr., BBR, 0.3 km SE Domzheritsy Vill., ruderal vegetation, 21 July 2000, Tussilago farfara L., on root collar, apt., S.V. Buga leg.; Miory Distr:: 1.5 km SW Pridorozh'e Vill., ruderal vegetation, 18 Aug. 2005, Tussilago farfara L., rhizomes, apt., gyn., S.V. Buga leg.; 2 km WSW Yurkovshchina Vill., ruderal vegetation, 2 July 2007, Tussilago farfara L., on petioles and lower side of leaves, apt., al., S.V. Buga leg.; Shumilino Distr., 2 km N Obol' (Obolj) Vill., dry meadow, 12 Sept. 2000, Tussilago farfara L., on root collar, apt., gyn., males, V.E. Jarigo leg.; Ushachi Distr., 2 km ESE Stai Vill., dry meadow, 21 July 2004, Tussilago farfara L., on root collar, apt., S.V. Buga leg.

Published information. Vitebsk Prov., Sharkoushchina (Szarkoreszczyzna) Distr., Bazylpol, 19 July 1924, Pyrus communis L. and Pyrus sp. (as Aphis Kochi Schontedon) (Fiedorowiczówna, 1930).

Anuraphis subterranea (Walker, 1852)

Specimens examined. Minsk Prov., Minsk Distr.: near Roshcha RWS, 14 July 1997, Heracleum sibiricum L, apt., al., S.V. Buga leg.; Priluki Vill., 14 July 1997, Pastinaca sativa L., on root collar, apt., al., S.V. Buga leg.

\section{Aspidaphis adjuvans (Walker, 1848)}

Specimens examined. Grodno (Grodna) Prov., Grodno (Grodna) Distr., Grodno, ruderal vegetation, 11 July 2004, Polygonum sp., on apical part of stem, al., S.V. Buga leg.

\section{Aulacorthum cylastis Börner, 1942}

Specimens examined. Gomel Prov., Zhitkovichi Distr., 2 km N Khvoensk Vill., Pripyat River valley, alder forest with species-rich grass cover, 12 July 1999, Rubus nessensis W. Hall, on apical part of stem, apt., S.V. Buga leg. 
Aulacorthum flavum F. P. Müller, 1958

Specimens examined. Brest Prov., $\mathrm{Ka}$ menets Distr., BPF, near Kamenyuki Vill., pine forest, 22 Sept. 2000, Vaccinium uliginosum L., on lower side of leaves, ovip. fem., S.V. Buga leg. Grodno (Grodna) Prov., Smorgon' Distr., 3.3 km SW Rachuny Vill., pine forest, 2 Sept. 2000, Vaccinium uliginosum L., on lower side of leaves, apt., S.V. Buga leg. Minsk Prov., Stolbtsy Distr., NBR, $1 \mathrm{~km}$ NE Kletishche Vill., pine forest, 14 July 2010, Vaccinium uliginosum L., on lower side of leaves, apt., S.V. Buga leg. Mogilev Prov., Osipovichi Distr., $0.5 \mathrm{~km}$ NE Verkhi RWS, pine forest, 19 June 2000, Vaccinium uliginosum L., on lower side of leaves, apt., S.V. Buga leg. Vitebsk Prov., Lepel' Distr., BBR, near Rozhno Vill., raised bog, 16 Aug. 1986, Vaccinium uliginosum L., on lower side of leaves, apt., S.V. Buga leg.

\section{Aulacorthum solani (Kaltenbach, 1843)}

Specimens examined. Brest Prov., Gantsevichi Distr., near Gantsevichi, swamped alder forest, 16 July 1991, Solanum dulcamara L., on apical part of stem, al., S.V. Buga leg. Minsk: CBG, arbortetum, 29 July 1987, Celastrus orbiculatus Thunb., on apical part of stem, apt., S.V. Buga leg.; CBG, rosarium, 21 June 1990, Rosa sp. hort. cv., on apical part of stem, apt., S.V. Buga leg.

\section{Brachycaudus helichrysi}

(Kaltenbach, 1843)

Specimens examined. Minsk, green stands, 1 July 2009, Leucanthemum maximum (Ramond) DC., on stem near inflorescences, apt., al., S.V. Buga leg. Minsk Prov., Molodechno Distr., $0.5 \mathrm{~km}$ NE Sychevichi Vill., dry meadow, 25 May 2006, Echium vulgare L., on lower side of leaves, apt., S.V. Buga leg. Vitebsk Prov., Postavy Distr., Polovo Vill., green stands, 20 July 2010, Leucanthemum maximum (Ramond) DC., on stem, apt., S.V. Buga leg.

Published information. Vitebsk Prov: Sharkovshchina (Szarkoweszczyzna) Distr.,
Bazylpol, 3 Sept. 1924, Prunus domestica L. (Fiedorowiczówna, 1930); same locality and date, but on Prunus insititia L. (as Aphis prunina) (Fiedorowiczówna, 1930).

\section{Brachycaudus spiraeae Börner, 1932}

Specimens examined. Brest Prov: Lyakhovichi Distr., near Zadvor'e Vill., green stands, 8 Aug. 2000, Spiraea salicifolia L., on leaves, S.V. Buga leg.; Ivatsevichi Distr., Byten' RWS, green stands, 23 Aug. 2000, Spiraea alba Du Roi, on lower side of leaves, S.V. Buga leg. Gomel Prov., Gomel Distr., Gomel, park, 5 Aug. 2000, Spiraea sp., on lower side of leaves, S.V. Buga leg. Minsk, green stands, 28 Aug. 1985, Spiraea salicifolia L., on lower side of leaves, apt., S.V. Buga leg. Minsk Prov., Borisov Distr., Borisov, green stands, 2 July 1987, Spiraea salicifolia $\mathrm{L}$., on lower side of leaves, apt., S.V. Buga leg. Vitebsk Prov.: Gorodok Distr., near Dubokray (Dubokraj) Vill., 18 June 2008, Spiraea sp., R. Rakauskas leg.; Shumilino Distr., Obol' (Obolj) Vill., green stands, 12 Sept. 2000, Spiraea alba Du Roi, on apical part of stem, S.V. Buga leg.

Published information. On Spiraea, without any faunal data (as Brachycaudus spiraeae (Oestl.)) (Gorlenko \& Pan'ko, 1972).

\section{Brachycaudus (Acaudus) divaricatae Shaposhnikov, 1956}

Specimens examined. Minsk Prov.: Kopyl' Distr: near Sloboda-Kuchinka Vill., green stands, 9 July 2010, Prunus divaricata Ledeb. hort. cv., on apices of stem, apt., S.V. Buga leg.; near Pesochnoe Vill., green stands, 9 July 2010, Prunus divaricata Ledeb. hort. cv., on apices of stem, apt., S.V. Buga leg.; Stolbtsy Distr., Stolbtsy, green stands, 9 July 2010, Prunus divaricata Ledeb. hort. cv., on apices of stem, apt., S.V. Buga leg. Vitebsk Prov.: Gorodok Distr., Gorodok, 17 June 2008, Prunus divaricata Ledeb. (as Prunus cerasifera Ehrh. in Rakauskas \& Buga, 2010) hort. cv., apt., R. Rakauskas leg.; Postavy Distr., Postavy, green 
stands, 20 July 2010, Prunus divaricata Ledeb. hort. cv., on apices of stem, apt., S.V. Buga leg.

\section{Brachycaudus (Acaudus) lychnidis (Linnaeus, 1758)}

Specimens examined. Minsk Prov., Kopyl' Distr., near Pesochnoe Vill., dry meadow, 10 July 2010, Silene borysthenica (Gruner) Walters, on stem near inflorescences, apt., S.V. Buga leg. Vitebsk Prov., Gorodok Distr., Rudnia (Rudnya, Rudnja) Vill., 18 June 2008, Silene vulgaris (Moench) Garcke, R. Rakauskas leg.

\section{Brachycaudus (Acaudus) populi} (del Guercio, 1911)

Specimens examined. Minsk Prov.: Molodechno Distr., near Udranka Vill., ruderal vegetation, 17 July 2007, Silene vulgaris (Moench) Garcke, on stem, apt., N.V. Leshchinskaya leg.; Smolevichi Distr., near Berezovaya Gora Vill., dry meadow, 21 June 2008, Silene sp., on apical part of stem and lower side of leaves, al., apt., S.V. Buga leg.

\section{Brachycaudus (Appelia) prunicola}

(Kaltenbach, 1843)

Published information. Prunus sp., without faunal data (Kharchanka, 1968).

\section{Brachycaudus (Appelia) tragopogonis} (Kaltenbach, 1843)

Specimens examined. Grodno (Grodna) Prov., Smorgon' Distr., near Soly RWS, ruderal vegetation, 24 June 2006, Tragopogon sp., on stem, apt., al., N.V. Leshchinskaya leg.

\section{Brachycaudus (Brachycaudina) aconiti} (Mordvilko, 1928)

Specimens examined. Vitebsk Prov., Beshenkovichi Distr., Beshenkovichi, 30 July 2007, Delphinium elatum L., on stem, apt., S.V. Buga leg.

\section{Brachycaudus (Prunaphis) cardui}

(Linnaeus, 1758)

Specimens examined. Minsk: CBG, roadside, ruderal vegetation, 14 July 2006 , Symphytum officinale L., apices of stem, apt., N.V. Leshchinskaya leg.; CBG, 25 July 2006, Inula sp., apices of stem, apt., N.V. Leshchinskaya leg.

Published information. Vitebsk Prov: Sharkovshchina (Szarkowszczyzna) Distr:: Bazylpol, 3 Sept. 1924, Prunus domestica L. (as Aphis pruni Koch) (Fiedorowiczówna, 1930); same locality and date, but Prunus insititia L. (Fiedorowiczówna, 1930).

\section{Brachycaudus (Prunaphis) mordvilkoi Hille Ris Lambers, 1931}

Specimens examined. Grodno (Grodna) Prov., Smorgon' Distr., near Soly RWS, ruderal vegetation, 15 June 2008, Echium vulgare L., on basal part of stem, al., N.V. Leshchinskaya leg. Minsk Prov., Molodechno Distr., near Udranka Vill., ruderal vegetation, 29 July 2006, Elymus repens L. (accidentally), on lower side of leaves, apt., N.V. Leshchinskaya leg.

Brevicoryne brassicae (Linnaeus, 1758)

Specimens examined. Minsk Prov., Minsk Distr., Priluki Vill., 7 Sept. 2002, Brassica oleracea L. hort. cv., on lower side of leaves, apt., al., S.V. Buga leg.

\section{Brachycorynella lonicerina}

(Shaposhnikov, 1952)

Specimens examined. Minsk Prov., Minsk Distr., near Priluki Vill., mixed forest, 27 Aug. 1985, Lonicera sp., on lower side of leaves, apt., S.V. Buga leg.

\section{Capitophorus elaeagni}

(del Guercio, 1894)

Specimens examined. Brest Prov., Zhabinka Distr., $0.2 \mathrm{~km}$ SE Malye Yakovchitsy Vill., strip-shaped forest patch, 17 Oct. 
2000, Hippophae rhamnoides L., on lower side of leaves, gyn., ovip. fem., S.V. Buga leg. Grodno (Grodna) Prov., Zel'va Distr., Pletyanichi RWS, strip-shaped forest patch, 14 Oct. 2000, Hippophae rhamnoides L., on lower side of leaves, gyn., S.V. Buga leg. Minsk Prov., Minsk Distr.: Priluki Vill., orchard, 28 Oct. 1999, Hippophae rhamnoides L., on lower side of leaves, gyn., males, ovip. fem. S.V. Buga leg.; same locality, 20 Oct. 2004, Hippophae rhamnoides L. hort. cv., on lower side of leaves, males, ovip. fem., S.V. Buga leg.

\section{Capitophorus hippophaes (Walker, 1852)}

Specimens examined. Brest Prov., Zhabinka Distr., $0.2 \mathrm{~km}$ SE Malye Yakovchitsy Vill., strip-shaped forest patch, 17 Oct. 2000, Hippophae rhamnoides L., on lower side of leaves, gyn., S.V. Buga leg. Grodno (Grodna) Prov., Smorgon' Distr., 3.3 km SW Rachuny Vill., forest road, 2 Sept. 2000, Persicaria hydropiper (L.) Delarbre, on lower side of leaves, apt., S.V. Buga leg. Minsk Prov.: Minsk Distr., Priluki Vill., orchard, 28 Oct. 1999, Hippophae rhamnoides L., on lower side of leaves, gyn., males, ovip. fem., S.V. Buga leg.; Nesvizh Distr., Khvoevo RWS, ruderal vegetation, 6 Sept. 2000, Persicaria lapathifolia (L.) Delarbre, on lower side of leaves, apt., S.V. Buga leg. Mogilev Prov., Klimovichi Distr., Klimovichi, green stands, 24 Aug. 2005, Hippophae rhamnoides L., on lower side of leaves, gyn., male, ovip. fem., S.V. Buga leg.

\section{Capitophorus pakansus}

Hottes et Frison, 1931

Specimens examined. Vitebsk Prov., Gorodok Distr., Gorodok, 7 June 2008, Inula helenium L., on lower side of leaves, apt., S.V. Buga leg.

\section{Capitophorus similis van der Goot, 1915}

Specimens examined. Minsk Prov., Minsk Distr., near Roshcha RWS, ruderal vegetation, 24 July 2006, Tussilago farfara L., on lower side of leaves, apt., N.V. Leshchinskaya leg.

\section{Cavariella aegopodii (Scopoli, 1763)}

Specimens examined. Minsk Prov., Borisov Distr., Borisov, Berezina river valley, green stands, 17 July 1987, Salix alba L., on lower side of leaves, apt., S.V. Buga leg.

\section{Cavariella archangelicae (Scopoli, 1763)}

Specimens examined. Minsk Prov., Minsk Distr., near Shchemyslitsa Vill., ruderal vegetation, 21 Aug. 1995, Pastinaca sylvestris L., on inflorescences, al., S.V. Buga leg.

\section{Cavariella cicutae (Koch, 1854)}

Specimens examined. Minsk, CBG, landscape park, 31 July 1986, Angelica sylvestris L., on inflorescence, apt., S.V. Buga leg. Minsk Prov., Volozhin Distr., NBR, hamlet Pechishche, floodplain meadow, 26 July 1987, Salix fragilis L., on lower side of leaves, apt., S.V. Buga leg.

\section{Cavariella konoi Takahashi, 1939}

Specimens examined. Vitebsk Prov., Gorodok Distr.: Zadrach'e (Zadrachje) Vill., 1 Aug. 2007, Salix fragilis L., apices of stem, al., S.V. Buga leg.; Rudnia (Rudnya, Rudnja) Vill., 18 June 2008, Salix fragilis L., apices of stem, apt., S.V. Buga leg.

\section{Cavariella pastinacae (Linnaeus, 1758)}

Specimens examined. Minsk Prov., Volozhin Distr., NBR, hamlet Pechishche, floodplain meadow, 25 July 1987, Salix triandra L., on lower side of leaves, apt., S.V. Buga leg. Vitebsk Prov., Gorodok Distr., Zadrach'e (Zadrachje) Vill., 2 June 2000, Salix pentandra L., on lower side of leaves, apt., S.V. Buga leg.

\section{Cavariella theobaldi \\ (Gillette et Bragg, 1918)}

Specimens examined. Gomel Prov., Zhitkovichi Distr., 2 km N Khvoensk Vill., bush growth at floodplain of the Pripyat River, 12 July 1999, Salix alba L., on apical part of 
stem, apt., S.V. Buga leg. Grodno (Grodna) Prov.: Mosty Distr., $1.5 \mathrm{~km} \mathrm{~S}$ Cherlena RWS, strip-shaped forest patch, 26 Aug. 2000, $\mathrm{He}$ racleum sibiricum L., on umbel, apt., al., S.V. Buga leg.; Oshmyany (Oshmjany) Distr., Oshmyany (Oshmjany) RWS, green stands, 4 Oct. 2005, Salix alba L., on lower side of leaves, males, ovip. fem., S.V. Buga leg. Minsk: CBG, arboretum, 16 Aug. 1985, Salix alba L., on lower side of leaves, al., S.V. Buga leg.; CBG, landscape park, 31 July 1986, Angelica sylvestris L., on inflorescences, apt., S.V. Buga leg.; CBG, landscape park, 18 Oct. 1996, Salix fragilis L., on apical part of stem, ovip. fem., S.V. Buga leg. Minsk Prov.: Minsk Distr.: near Priluki Vill., bushes, 10 Aug. 1987, Salix aurita L., on lower side of leaves, apt., al., S.V. Buga leg.; same locality, but mixed forest, 4 Oct. 1987, Salix caprea $\mathrm{L}$., on lower side of leaves, ovip. fem., S.V. Buga leg.; near Pomyslishche RWS, ruderal vegetation, 21 Aug. 1995, Pastinaca sylvestris Mill., on inflorescences, al., S.V. Buga leg.; near Ratomka Vill., strip-shaped forest patch, 1 July 1999, Salix cinerea L., on apical part of stem, apt., al., S.V. Buga leg.; $0.5 \mathrm{~km}$ SW Pomyslishche RWS, strip-shaped forest patch, 2 Oct. 1999, Salix repens L., on apical part of stem, ovip. fem., S.V. Buga leg.; near Pomyslishche RWS, ruderal vegetation, 16 July 2000, Pastinaca sylvestris Mill., on stem, apt., al., S.V. Buga leg.; Pukhovichi Distr., 2 km SW Dem'yanovka Vill., mixed forest, 16 June 2000, Salix cinerea L., on apical part of stem, al., S.V. Buga leg.; Starye Dorogi Distr., 3 km W Falichi RWS, forest glade, 3 Aug. 2000, Heracleum sibiricum L., on umbel, apt., S.V. Buga leg.; Volozhin Distr., NBR, hamlet Pechishche, 18 Oct. 1985, Salix fragilis L., on lower side of leaves, apt., gyn., males, S.V. Buga leg. Vitebsk Prov.: Gorodok Distr., Rudnia (Rudnya, Rudnja) Vill., 18 June 2008, Salix fragilis L., apical part of stem, apt., S.V. Buga leg.; Lepel' Distr.: $1 \mathrm{~km}$ W Domzheritsy Vill., mixed forest, 4 June 1990, Salix cinerea L., on apical part of stem, al., S.V. Buga leg.; Domzheritsy Vill., wasteland, 21 July 2000, Heracleum sibiricum L., on umbels, apt., S.V. Buga leg.; 0.3 km SE Domzheritsy Vill., floodplain meadow, 21 July 2000 , Salix cinerea L., on apical part of stem, apt., al., S.V. Buga leg.; Liozno Distr., near Liozno RWS, dry meadow, 20 June 2008, Pastinaca sylvestris Mill., on apical part of stem, apt., al., S.V. Buga leg.

\section{Ceruraphis eriophori (Walker, 1848)}

Specimens examined. Brest Prov.: Baranovichi Distr., $1.5 \mathrm{~km}$ SSE Melyakhovichi Vill., mixed forest, 5 May 2000, Viburnum opulus L., on lower side of leaves, em., S.V. Buga leg.; Kamenets Distr., BPF, Kamenyuki Vill., green stands, 14 Oct. 1999, Viburnum opulus L., on lower side of leaves, ovip. fem., S.V. Buga leg. Minsk Prov., Minsk Distr., near Priluki Vill., floodplain forest, 4 Oct. 1987, Viburnum opulus L., on lower side of leaves, ovip. fem., S.V. Buga leg. Mogilev Prov., Gorki Distr., 0.8 km N Kukshinovo Vill., spruce-dominated forest, 10 Oct. 2000, Viburnum opulus L., on lower side of leaves, ovip. fem., S.V. Buga leg.

\section{Chaetosiphon (Pentatrichopus) tetrarhodum (Walker, 1849)}

Specimens examined. Minsk: CBG, arboretum, 9 Aug. 1985, Rosa x waitziana Tratt., on lower side of leaves, apt., S.V. Buga leg.; green stands, 17 Sept. 1998, Rosa rugosa Thunb., on lower side of leaves, apt., S.V. Buga leg.; green stands, 19 May 2001, Rosa rugosa Thunb., on buds, apt., S.V. Buga leg. Minsk Prov., Minsk Distr., near Shchemyslitsa Vill., arboretum, 21 Aug. 1986, Rosa sp., on lower side of leaves, apt., S.V. Buga leg. Vitebsk Prov., Postavy Distr., Postavy, green stands, 20 July 2010, Rosa rugosa Thunb., on lower side of leaves, apt., S.V. Buga leg.

Coloradoa artemisiae (del Guercio, 1913)

Specimens examined. Minsk, ruderal vegetation, 15 June 1996, Artemisia absinthium L., on lower side of leaves, apt., Yu.V. Safonova leg. 


\section{Corylobium avellanae (Schrank, 1801)}

Specimens examined. Brest Prov.: Kamenets Distr., BPF, near Kamenyuki Vill., edge of mixed forest, 13 Oct. 1999, Corylus avellana L., on lower side of leaves, males, ovip. fem., S.V. Buga leg.; Lyakhovichi Distr., 0.6 km NNE Zadvor'e Vill., pine forest, 8 Aug. 2000, Corylus avellana L., on apical part of stem, apt., S.V. Buga leg. Grodno (Grodna) Prov., Smorgon' Distr., 2 km SW Rachuny Vill., hazel growth, 2 Sept. 2000, Corylus avellana L., on lower side of leaves, apt., S.V. Buga leg. Minsk, CBG, landscape park, 25 June 1987, Corylus avellana L., on apical part of stem, S.V. Buga leg. Minsk Prov.: Borisov Distr., near Leshchiny Vill., mixed forest, 25 June 1987, Corylus avella$n a$ L., on apical part of stem, apt., al., S.V. Buga leg.; Dzerzhinsk Distr., 0.1 km S Asino RWS, drained forested terrain, 7 July 2000 , Corylus avellana L., on apical part of stem, apt., S.V. Buga leg.; Pukhovichi Distr., $1 \mathrm{~km}$ SW Dem'yanovka Vill., pine forest, 16 June 2000, Corylus avellana L., on apical part of stem, apt., S.V. Buga leg.; Starye Dorogi Distr., $3 \mathrm{~km} \mathrm{~W} \mathrm{Falichi} \mathrm{RWS,} \mathrm{hornbeam}$ forest, 3 Aug. 2000, Corylus avellana L., on apical part of stem, apt., S.V. Buga leg.; Stolbtsy Distr., $0.3 \mathrm{~km}$ E Kolosovo RWS, pine forest, 27 June 2000, Corylus avellana L., on apical part of stem, apt., S.V. Buga leg.; Uzda Distr., 2 km SE Mezinovka RWS, spruce-dominated forest, 27 June 2000, Corylus avellana $\mathrm{L}$., on apical part of stem, apt., S.V. Buga leg. Vitebsk Prov.: Gorodok Distr: $1.5 \mathrm{~km}$ NE Makhalovo Vill., birch forest, 4 June 2000, Corylus avellana L., on apical part of stem, al., S.V. Buga leg.; 1.5 km WSW Bolotnitsa (Bolotnica) Vill., pine forest, 6 June 2000, Corylus avellana L., on apical part of stem, al., S.V. Buga leg.; Surmino, 10 July 2000, Corylus avella$n a$ L., on apical part of stem, al., S.V. Buga leg.; Lepel' Distr., BBR, 1 km W Domzheritsy Vill., pine forest, 11 June 2000, Corylus avellana $\mathrm{L}$., on apical part of stem, apt., D.L. Petrov leg.

\section{Cryptomyzus galeopsidis}

(Kaltenbach, 1843)

Specimens examined. Minsk Prov.: Minsk Distr., Priluki Vill., 31 July 1996, Galeopsis bifida Boenn., on lower side of leaves, apt., al., S.V. Buga leg.; Starye Dorogi Distr., 3 km W Falichi RWS, ruderal vegetation, 6 Aug. 2000, Galeopsis bifida Boenn., on lower side of leaves, al., S.V. Buga leg. Mogilev Prov., Krugloe Distr., $1.5 \mathrm{~km}$ SW Staroe Polessie Vill., oat field, 29 Aug. 2000, Galeopsis bifida Boenn., on lower side of leaves, apt., S.V. Buga leg. Vitebsk Prov.: Lepel' Distr., BBR, 3 km SW Perekhodtsy Vill., sprucedominated forest, 8 July 2004, Ribes nigrum L., on lower side of leaves, apt., al., D.L. Petrov leg.; Orsha Distr., 7 km W Ivan'kovo Vill., black alder wood in a brook valley, 27 Sept. 2000, Ribes lucidum Kit., on lower side of leaves, gyn., S.V. Buga leg.

Cryptomyzus leonuri Bozhko, (1957) 1961

Specimens examined. Gomel Prov., Dobrush Distr., $1 \mathrm{~km}$ NE Dobrush RWS, pine forest with species-rich grass cover, 28 Sept. 2007, Leonurus quinquelobatus Gilib., on lower side of leaves, apt., S.V. Buga leg. Vitebsk Prov.: Beshenkovichi Distr., near Zorniki Vill., ruderal vegetation, 17 June 2008, Leonurus quinquelobatus Gilib., on lower side of leaves, apt., S.V. Buga leg.

\section{Cryptomyzus ribis (Linnaeus, 1758)}

Specimens examined. Minsk Prov., Minsk Distr., Kryzhovka Vill., orchard, 21 May 2000, Ribes rubrum L. hort. cv., on lower side of leaves, apt., A.A. Mityanin leg. Vitebsk Prov., Gorodok Distr., Ezerishche (Jezerishche), 19 June 2008, Ribes sp. hort. cv., apt., R. Rakauskas leg.

\section{Diuraphis (Holcaphis) frequens \\ (Walker, 1848)}

Specimens examined. Minsk Prov., Starye Dorogi Distr., Yazyl', 8 July 1929, Elymus sp., apt., al., A.K. Mordvilko leg. 
Dysaphis affinis (Mordvilko, 1928)

Specimens examined. Vitebsk Prov., Gorodok Distr., Zadrach'e (Zadrachje) Vill., 18 June 2008, Malus domestica Borkh. hort. cv., R. Rakauskas leg.

\section{Dysaphis angelicae (Koch, 1854)}

Specimens examined. Grodno (Grodna) Prov., Iv'e (Iv'ye, Iv'evsk) Distr., $0.6 \mathrm{~km} \mathrm{~S}$ Yahimovshchina Vill., spruce-donminated forest, 27 Aug. 2010, Angelica sylvestris L., axils of leaves, apt., S.V. Buga leg.

Dysaphis anthrisci Börner, 1950

Specimens examined. Minsk Prov., Stolbtsy Distr., NBR, near hamlet Voynilovshchina, ruderal vegetation, 14 July 2010, Anthriscus sylvestris (L.) Hoffm., petioles of rosette leaves, apt., S.V. Buga leg.

\section{Dysaphis apiifolia petroselini \\ (Börner, 1950)}

Specimens examined. Minsk Prov., Minsk Distr., Pomyslishche RWS, green stands, 21 June 1997, Crataegus monogyna Jacq., in leaf gall, fund., em., leg S.V. Buga.

\section{Dysaphis brancoi (Börner, 1950)}

Specimens examined. Minsk Prov., Molodechno Distr., near Udranka Vill., dry meadow, 19 June 2004, Malus praecox (Pall.) Borkh., in leaf gall, em., S.V. Buga leg. Vitebsk Prov., Gorodok Distr., Zadrach'e (Zadrachje) Vill., 5 Aug. 2007, Valeriana officinalis L., on root collar, apt., S.V. Buga leg.

Dysaphis devecta (Walker, 1849)

Specimens examined. Minsk Prov., Pukhovichi Distr., $0.3 \mathrm{~km} \mathrm{SW} \mathrm{Tal'ka} \mathrm{RWS,} \mathrm{edge}$ of mixed forest, 16 June 2000, Malus sylvestris Mill., in leaf gall, apt., S.V. Buga leg.

\section{Dysaphis hirsutissima (Börner, 1940)}

Specimens examined. Gomel Prov., Dobrush Distr., 1 km NE Dobrush RWS, ru- deral vegetation, 22 Aug. 2009, Anthriscus sylvestris (L.) Hoffm., petioles of rosette leaves, apt., S.V. Buga leg. Minsk Prov., Kopyl' Distr., near Pechurany Vill., mixed forest, 10 July 2010, Anthriscus sylvestris (L.) Hoffm., on axils of rosette leaves, apt., S.V. Buga leg. Vitebsk Prov.: Beshenkovichi Distr:: near Beshenkovichi, mixed forest, 30 July 2007, Anthriscus sylvestris (L.) Hoffm., on root collar and base of leaf petioles, apt., S.V. Buga leg.; same locality, but ruderal vegetation, 5 Aug. 2007, Anthriscus sylvestris (L.) Hoffm., on root collar and base of leaf petioles, apt., S.V. Buga leg.; Gorodok Distr., near Marchenki Vill., ruderal vegetation, 29 Aug. 2002, Anthriscus sylvestris (L.) Hoffm., on root collar, apt., S.V. Buga leg.; Laptevka Vill., 19 June 2008, Anthriscus sylvestris (L.) Hoffm., apt., R. Rakauskas leg.; Liozno Distr., Velikoe Selo Vill., ruderal vegetation, 20 June 2008, Anthriscus sylvestris (L.) Hoffm., on petioles of rosette leaves, apt., S.V. Buga leg.; Vitebsk Distr., near Babinichi Vill., 20 June 2008, Anthriscus sylvestris (L.) Hoffm., on root collar, apt., S.V. Buga leg.

\section{Dysaphis lauberti (Börner, 1940)}

Specimens examined. Minsk Prov., Kopyl' Distr., near Pechurany Vill., mixed forest, 10 July 2010, Heracleum sibiricum L., axils of leaves, apt., S.V. Buga leg.

\section{Dysaphis radicola (Mordvilko, 1897)}

Specimens examined. Minsk, orchard, 16 June 1997, Malus domestica Borkh. hort. cv., in leaf gall, em., S.V. Buga leg.

Dysaphis ranunculi (Kaltenbach, 1843)

Specimens examined. Minsk, green stands, 12 June 1997, Crataegus sp., in leaf gall, em., S.V. Buga leg. Minsk Prov., Minsk Distr., near Roshcha RWS, 15 June 1997, Crataegus monogyna Jacq., in leaf gall, fund., em., S.V. Buga leg.

Published information. Grodno (Grodna) Prov., Oshmyany (Oshmjany) Distr., Oshmy- 
any (Oshmjany), 1 Sept. 1932, Crataegus sp. (as Myzus oxyacanthae Koch and Anuraphis ranunculi Kalf.) (Perepeczko-Baumanowa, 1934).

Fiedorowiczówna (1930) recorded Anuraphis ranunculi Kalf. and Myzus oxyacanthae Koch from Vitebsk Prov:: Sharkovshchina (Szarkozeszczyzna) Distr., Bazylpol, 19 July 1924, Pyrus communis L. (Fiedorowiczówna, 1930); Bazylpol, 23 July 1924, Crataegus monogyna Jacq. (Fiedorowiczówna, 1930); vill. Radyuki, 11 Aug. 1924, Crataegus sp. (Fiedorowiczówna, 1930). However, D. ranunculi is a dioecious species, which under the condition of Belarus completes its migration from primary to secondary host by the end of June; therefore the probability of finding this species in July and in the first half of August on Crataegus, and even more so on Pyrus (not a host plant) is rather small. Apparently in this case we have some other species of aphids reported under these names.

\section{Dysaphis (Pomaphis) plantaginea} (Passerini, 1860)

Specimens examined. Minsk: green stands, 12 July 1997, Malus domestica Borkh. hort. cv., on apical part of stem and lower side of leaves, apt., al., S.V. Buga leg.; 13 July 1997, Malus domestica Borkh. hort. cv., on apical part of stem and lower side of leaves, apt., al., S.V. Buga leg.; green stands, 16 July 1997, Malus domestica Borkh. hort. cv., on apical part of stem and lower side of leaves, apt., al., S.V. Buga leg.; orchard, 17 July 1997, Malus domestica Borkh. hort. cv., on apical part of stem and lower side of leaves, apt., al., S.V. Buga leg. Minsk Prov.: Dzerzhinsk Distr., $0.1 \mathrm{~km}$ S Asino RWS, drained forested terrain, 7 July 2000, $M a$ lus domestica Borkh. hort. cv., on lower side of leaves, apt., al., S.V. Buga leg.; Volozhin Distr., NBR, hamlet Pechishche, pine forest, 25 July 1987, Malus sylvestris Mill., on apical part of stem and lower side of leaves, apt., al., S.V. Buga leg. Vitebsk Prov.: Gorodok Distr., Zadrach'e (Zadrachje) Vill.,
18 June 2008, Malus domestica Borkh. hort. cv., R. Rakauskas leg.; Lepel' Distr., BBR, Domzheritsy Vill., orchards, 21 July 2000, Malus domestica Borkh. hort. cv., on lower side of leaves, al., S.V. Buga leg.

Published information. Fiedorowiczówna (1930) had registered Myzus mali Ferrari and Myzus discrepans Koch from Pyrus sp. and Pyrus communis L. (Vitebsk Prov., Sharkovshchina (Szarkoweszczyzna) Distr., Bazylpol, 7 July 1924). These species have been synonymised with Dysaphis (Pomaphis) plantaginea (Passerini, 1860), but its feeding on pear looks doubtful.

\section{Dysaphis (Pomaphis) sorbi}

(Kaltenbach, 1843)

Specimens examined. Minsk: green stands, 2 July 1997, Sorbus aucuparia L., in rolled leaves, apt., S.V. Buga leg.; strip-shaped forest patch, 15 June 2001, Sorbus aucuparia L., in rolled leaves, al., S.V. Buga leg. Minsk Prov.: Lyuban' Distr., near Oboz Vill., green stands, 24 Sept. 2004, Sorbus aucuparia L., on apical part of stem, gyn., S.V. Buga leg.; Minsk Distr., Priluki Vill., 18 June 1929, apt., em., A.K. Mordvilko leg.; Volozhin Distr., 1 km SW Kaldyki Vill., 18 June 1999, Sorbus aucuparia L., on apical part of stem, apt., S.V. Buga leg. Mogilev Prov.: Osipovichi Distr., 0.5 km NE Verkhi RWS, pine forest, 19 June 2000, Sorbus aucuparia L., in rolled leaves, apt., S.V. Buga leg.; Slavgorod Distr., $1.5 \mathrm{~km}$ NNW Zavod-Virovaya Vill., pine forest, 24 Aug. 2005, Sorbus aucuparia L., on apical part of stem, al., S.V. Buga leg. Vitebsk Prov., Gorodok Distr.: Surmino Vill., 10 July 2000, Sorbus aucuparia L., on apical part of stem, apt., S.V. Buga leg.; Ezerishche (Jezerishche), green stands, 19 June 2008, Sorbus aucuparia L., in rolled leaves, apt., S.V. Buga leg.; Lepel' Distr., BBR, near Domzheritsy Vill., pine forest, 16 Aug. 1986, Sorbus aucuparia L., on apical part of stem, apt., S.V. Buga leg.; Rossony Distr., near lake Glubochino, pine forest, 3 July 2007, Sorbus aucuparia L., on apical part of stem, apt., al., S.V. Buga leg. 
Published information. Brest Prov., on Sorbus aucuparia L. (as Dentatus sorbi Kalt.) (Pan'ko, 1969). Brest and Gomel Prov. (as Dentatus sorbi Kalt.) (Pan'ko, 1969). Minsk, CBG, on Sorbus matsumurana (Makino) Koehne (as Dentatus sorbi Kalt.) (Gorlenko \& Pan'ko, 1967, 1972).

\section{Elatobium abietinum (Walker, 1849)}

Specimens examined. Minsk, green stands, 5 July 1999, Picea pungens Engelm., on needles, apt., V.E. Jarigo leg. Minsk Prov., Starye Dorogi Distr., Yazyl', 10 July 1929, Picea sp., apt., A.K. Mordvilko leg.

\section{Ericaphis latifrons (Börner, 1942)}

Specimens examined. Vitebsk Prov., Shumilino Distr., 4 km N Obol' (Obolj) RWS, raised bog, 12 Sept. 2000, Empetrum nigrum L., on apical part of stem, apt., V.E. Jarigo leg.

\section{Hayhurstia atriplicis (Linnaeus, 1761)}

Specimens examined. Grodno (Grodna) Prov., Grodno (Grodna) Distr., Grodno (Grodna), ruderal vegetation, 10 July 2004, Chenopodium album L., in leaf gall, al., S.V. Buga leg. Minsk, CBG, ruderal vegetation, 29 July 1985, Chenopodium album L., in leaf gall, apt., S.V. Buga leg. Minsk Prov., Molodechno Distr., near Udranka Vill., ruderal vegetation, 17 July 1997, Chenopodium album L., in leaf gall, apt., S.V. Buga leg. Mogilev Prov., Slavgorod Distr., $1.2 \mathrm{~km}$ ESE Novaya Sloboda Vill., ruderal vegetation, 24 Aug. 2005, Chenopodium glaucum L., in leaf gall, apt., males, S.V. Buga leg. Vitebsk Prov.: Polotsk Distr., Novopolotsk, green stands, 2 July 2007, Chenopodium album L., in leaf gall, apt., al., S.V. Buga leg.; Vitebsk Distr., Vitebsk, ruderal vegetation, 30 July 2007, Chenopodium sp., in leaf gall, apt., S.V. Buga leg.

\section{Hyadaphis foeniculi (Passerini, 1860)}

Specimens examined. Minsk Prov., Minsk Distr., 0.4 km S Prilukskaya Sloboda Vill., ruderal vegetation, 5 July 2004, Anthriscus sylvestris (L.) Hoffm., on inflorescences, apt., al., S.V. Buga leg. Vitebsk Prov.: Gorodok Distr., Zadrach'e (Zadrachje) Vill., 4 July 1996, Aegopodium podagraria L., on inflorescences, S.V. Buga leg.; Lepel' Distr., BBR, 3 km SW Perekhodtsy Vill., oak-dominated forest with species-rich grass cover, 8 July 2004, Anthriscus sylvestris (L.) Hoffm., on inflorescences, apt., al., S.V. Buga leg.

Published information. Vitebsk Prov: Braslav Distr., Druya (Druja), 22 July 1924, Lonicera tatarica L. (as Siphocoryne xylostei Schrank.) (Fiedorowiczówna, 1930); Miory Distr., Krichevo (Krycewicze), 22 July 1924, Lonicera tatarica L. (as Siphocoryne xylostei Schrank.) (Fiedorowiczówna, 1930).

Hyadaphis passerinii (del Guercio, 1911)

Specimens examined. Grodno (Grodna) Prov., Iv'e (Iv'ye, Iv'evsk) Distr., $0.75 \mathrm{~km}$ WSW Gav'ya RWS, strip-shaped forest patch, 9 Sept. 2000, Lonicera tatarica L., on apical part of stem, apt., S.V. Buga leg.

\section{Hyadaphis tataricae Aizenberg, 1935}

Specimens examined. Grodno (Grodna) Prov.: Lida Distr., Neman RWS, green stands, 31 Aug. 2000, Lonicera tatarica L., on lower side of leaves, al., S.V. Buga leg.; Oshmyany (Oshmjany) Distr., Oshmyany (Oshmjany) RWS, green stands, 4 Oct. 2005, Lonicera tatarica L., on lower side of leaves, gyn., S.V. Buga leg. Minsk: green stands, 14 July 1986, Lonicera tatarica L., in leaf gall, apt., S.V. Buga leg.; green stands, 11 Nov. 1997, Lonicera tatarica L., on lower side of leaves, ovip. fem., S.V. Buga leg. Minsk Prov.: Borisov Distr., Borisov, green stands, 2 July 1987, Lonicera tatarica L., in leaf gall, apt., S.V. Buga leg.; Minsk Distr., Zhdanovichi Vill., green stands, 21 Sept. 1999, Lonicera tatarica L., on lower side of leaves, gyn., ovip. fem., S.V. Buga leg. Vitebsk Prov.: Shumilino Distr., $1 \mathrm{~km}$ WNW Obol' (Obolj) RWS, strip-shaped forest patch, 12 Sept. 2000, Lonicera tatarica L., 
on lower side of leaves, apt., S.V. Buga leg.; Tolochin Distr., Slavnoe RWS, green stands, 29 Aug. 2000, Lonicera tatarica L., on apical part of stem, al., S.V. Buga leg.

\section{Hydaphias molluginis Börner, 1939}

Specimens examined. Minsk Prov.: Minsk Distr., near Zabolot'e Vill., meadow, 20 June 2006, Galium sp., on stem, al., N.V. Leshchinskaya leg.; Molodechno Distr., near Udranka Vill., dry meadow, 25 June 2006 and 22 May 2008, Galium mollugo L., on apical part of stem, apt., S.V. Buga leg. Vitebsk Prov., Gorodok Distr: near Dubokray (Dubokraj), 18 June 2008, Galium mollugo L., apt., R. Rakauskas leg.; Laptevka Vill., 19 June 2008, Galium mollugo L., apt., R. Rakauskas leg.

\section{Hyperomyzus lactucae (Linnaeus, 1758)}

Specimens examined. Minsk, 21 June 1929, Ribes nigrum L., on lower side of leaves, apt., em., A.K. Mordvilko leg. Minsk Prov.: Minsk Distr., Priluki Vill., orchard, 21 Oct. 2000, Ribes nigrum L., on lower side of leaves, gyn., S.V. Buga leg.; Smolevichi Distr., near Berezovaya Gora Vill., ruderal vegetation, 21 June 2008, Sonchus arvensis L., on apical part of stem, apt., al., D.L. Petrov leg.

\section{Hyperomyzus pallidus}

Hille Ris Lambers, 1935

Specimens examined. Minsk Prov.: Minsk Distr., near Ptich' RWS, ruderal vegetation, 26 July 2006, Sonchus arvensis L., on stem, apt., N.V. Leshchinskaya leg.

\section{Hyperomyzus (Hyperomyzella) rhinanthi} (Schouteden, 1903)

Specimens examined. Mogilev Prov:: Gorki Distr., near Zubry RWS, pine forest, 20 June 2008, Ribes nigrum L., on lower side of leaves, em., S.V. Buga leg.

\section{Impatientinum asiaticum asiaticum Nevsky, 1929}

Specimens examined. Minsk, CBG, landscape park, 16 Sept. 1997, Impatiens par- viflora DC., on flowers and petioles, apt., S.V. Buga leg. Minsk Prov., Minsk Distr., near Roshcha RWS, 24 July 2006, Impatiens parviflora DC., on flowers, apt., al., N.V. Leshchinskaya leg. Vitebsk Prov., Gorodok Distr., Bolotnitsa (Bolotnica) Vill., 6 Aug. 2007, Impatiens parviflora DC., on flowers and pedicels, apt., S.V. Buga leg.

\section{Liosomaphis berberidis}

(Kaltenbach, 1843)

Specimens examined. Gomel Prov., Gomel Distr., Gomel, park, 5 Aug. 2000, Berberis vulgaris L., on lower side of leaves, apt., S.V. Buga leg. Grodno (Grodna) Prov., Lida Distr., $1 \mathrm{~km}$ NE Neman RWS, bushes, 31 Aug. 2000, Berberis vulgaris L., on lower side of leaves, apt., S.V. Buga leg. Minsk: CBG, arboretum, 18 July 1986, Berberis vulgaris L. and Berberis thunbergii DC., on lower side of leaves, apt., S.V. Buga leg.; arboretum, 1 Aug. 1986, Berberis aquifolium Pursh., on lower side of leaves, apt., S.V. Buga leg. Minsk Prov., Nesvizh Distr., $0.4 \mathrm{~km} \mathrm{~W}$ Lipa RWS, pine forest, 6 Sept. 2000, Berberis vulgaris L., on lower side of leaves, apt., S.V. Buga leg. Vitebsk Prov., Lepel' Distr., BBR, Domzheritsy Vill., green stands, 21 July 2000, Berberis vulgaris L., on lower side of leaves, apt., S.V. Buga leg.

\section{Lipaphis pseudobrassicae (Davis, 1914)}

Specimens examined. Brest Prov., Gantsevichi Distr., near Gantsevichi, forest road, 16 July 1991, Erysimum sp., on inflorescence, apt., S.V. Buga leg. Minsk Prov., Molodechno Distr:: near Udranka Vill., meadow, 15 May 2004, Alliaria petiolata (Bieb.) Cavara et Grande, on inflorescence, apt., al., S.V. Buga leg.; same locality and date, but dry meadow, 22 June 2006, Erysimum cheiranthoides L., apices of stem, apt., N.V. Leshchinskaya leg.

Published information. Vitebsk Prov. BBR (as Lipaphis erysimi Kalt.) (Buga, 1989). 
Lipaphis turritella (Wahlgren, 1938)

Specimens examined. Minsk Prov., Molodechno Distr., nearUdranka Vill., dry meadow, 22 June 2006, Brassicaceae, on stems and inflorescences, apt., N.V. Leshchinskaya leg. Vitebsk Prov., Gorodok Distr.: near Dubokray (Dubokraj) Vill., 18 June 2008, Arabis glabra (L.) Bernh., apt., R. Rakauskas leg.; Sharino Vill., 19 June 2008, Arabis glabra (L.) Bernh., apt., R. Rakauskas leg.

\section{Longicaudus trirhodus (Walker, 1849)}

Specimens examined. Grodno (Grodna) Prov.: Lida Distr., 1 km NE Neman RWS, dry meadow, 31 Aug. 2000, Rosa villosa L., on lower side of leaves, al., S.V. Buga leg.; Smorgon' Distr., $3.3 \mathrm{~km}$ NNE Soly RWS, pine forest, 2 Sept. 2000, Rosa sp., on lower side of leaves, gyn., S.V. Buga leg.

\section{Macrosiphoniella absinthii} (Linnaeus, 1758)

Specimens examined. Minsk Prov., Molodechno Distr., near Udranka Vill., dry meadow, 29 June 2006, Artemisia campestris L., apices of stem, apt., N.V. Leshchinskaya leg. Vitebsk Prov., Beshenkovichi Distr., near Zorniki Vill., ruderal vegetation, 17 June 2008, Artemisia absinthium L., on lower side of leaves, apt., S.V. Buga leg.

\section{Macrosiphoniella artemisiae}

(Boyer de Fonscolombe, 1841)

Specimens examined. Vitebsk Prov., Gorodok Distr., Zadrach'e (Zadrachje) Vill., 31 July 2007, Artemisia vulgaris L., on lower side of leaves, apt., S.V. Buga leg.

\section{Macrosiphoniella austriacae Bozhko, 1961}

Specimens examined. Brest Prov., Gantsevichi Distr., near Gantsevichi, dry meadow, 11 July 1996, Artemisia austriaca Jacq., on lower side of leaves, apt., S.V. Buga leg.

\section{Macrosiphoniella dimidiata Börner, 1942}

Specimens examined. Gomel Prov., Zhlobin Distr., near Zhlobin, dry meadow, 27 June 2008, Artemisia campestris L., on apical part of stem, apt., N.V. Leshchinskaya leg. Minsk Prov., Smolevichi Distr., nearby Berezovaya Gora Vill., dry meadow, 21 June 2008, Artemisia vulgaris L., on lower side of leaves, apt., S.V. Buga leg. Vitebsk Prov.: Gorodok Distr., Laptevka, 19 June 2008, Artemisia campestris L., apt., S.V. Buga leg.; Liozno Distr., near Liozno RWS, dry meadow, 20 June 2008, Artemisia campestris L., on apical part of stem, apt., S.V. Buga leg.

\section{Macrosiphoniella millefolii \\ (de Geer, 1773)}

Specimens examined. Minsk Prov., Minsk Distr.: near Priluki Vill., strip-shaped forest patch, 1 Nov. 1993, Achillea millefolium L. s.l., on lower side of leaves, ovip. fem., S.V. Buga leg.; nearby Ptich' RWS, ruderal vegetation, 20 June 2006, Achillea millefolium L. s.l., on lower side of leaves, apt., N.V. Leshchinskaya leg. Vitebsk Prov: Gorodok Distr:: Makhalovo Vill., 3 June 2000, Achillea millefolium L. s.l., on lower side of leaves, apt., S.V. Buga leg.; near Osmoto lake, 19 June 2008, Achillea millefolium L. s.l., on lower side of leaves, apt., al., S.V. Buga leg. Lepel' Distr., BBR, 3 km SW Perekhodtsy Vill., meadow, 8 July 2004, Achillea millefolium L. s.l., on inflorescence, al., S.V. Buga leg.; Vitebsk Distr., Vitebsk, ruderal vegetation, 29 July 2006, Achillea millefolium L. s.l., on stem, apt., N.V. Leshchinskaya leg.

\section{Macrosiphoniella sanborni (Gillette, 1908)}

Specimens examined. Minsk, Loshitsa, arboretum, flowerbed, 12 Oct. 1987, Chrysanthemum $\times$ koreanum Makai hort. cv., on lower side of leaves, apt., S.V. Buga leg. 


\section{Macrosiphoniella tanacetaria}

(Kaltenbach, 1843)

Specimens examined. Vitebsk Prov., Verkhnedvinsk Distr., Lunacharskoe Vill., 22 Aug. 2006, Tanacetum vulgare L., apt., N.V. Leshchinskaya leg.

\section{Macrosiphoniella usquertensis}

Hille Ris Lambers, 1935

Specimens examined. Vitebsk Prov., Lepel' Distr., BBR, 3 km SW Perekhodtsy Vill., floodplain meadow, 8 July 2004, Achillea salicifolia Besser, on apical part of stem, apt., S.V. Buga leg.

\section{Macrosiphoniella (Phalangomyzus) \\ oblonga (Mordvilko, 1901)}

Specimens examined. Brest Prov. Gantsevichi Distr., near Gantsevichi, dry meadow, 11 July 1996, Artemisia absinthium L., on lower side of leaves, apt., S.V. Buga leg.

\section{Macrosiphoniella (Ramitrichophorus) janckei Börner, 1939}

Specimens examined. Minsk Prov. Molodechno Distr., near Udranka Vill., dry meadow, 6 July 1995 and 12 July 2007, $\mathrm{He}$ lichrysum arenarium (L.) Moench, on inflorescence, apt., S.V. Buga leg.

\section{Macrosiphum albifrons Essig, 1911}

Specimens examined. Mogilev Prov. Osipovichi Distr., near Verkhi RWS, mixed forest, 1 July 2008, Lupinus polyphyllus L., on lower side of leaves, al., N.V. Leshchinskaya leg.

\section{Macrosiphum cholodkovskyi \\ (Mordvilko, 1909)}

Specimens examined. Minsk Prov.: Minsk Distr., near Priluki Vill., floodplain meadow, 11 Aug. 1985, Filipendula ulmaria (L.) Maxim., on lower side of leaves, apt., S.V. Buga leg.; Volozhin Distr., NBR, hamlet Pech- ishche, floodplain meadow, 26 July 1987 , Filipendula ulmaria (L.). Maxim., on lower side of leaves, apt., S.V. Buga leg. Vitebsk Prov., Lepel' Distr., BBR, near Domzheritsy Vill., floodplain meadow, 4 June 2010, Filipendula ulmaria (L.). Maxim., on apical part of stem, apt., O.L. Nesterova leg.

\section{Macrosiphum daphnidis Börner, 1940}

Specimens examined. Brest Prov., $\mathrm{Ka}$ menets Distr., BPF, near Kamenyuki Vill., spruce-dominated forest, 22 Sept. 2000, Daphne mezereum L., on lower side of leaves, ovip. fem., S.V. Buga leg. Minsk Prov. Minsk Distr., near Korolev Stan Vill., spruce-dominated forest, 28 Sept. 1990, Daphne mezereum L., on lower side of leaves, ovip. fem., S.V. Buga leg. Vitebsk Prov., Lepel' Distr., BBR, $2 \mathrm{~km}$ SSE hamlet Zaboen'e, spruce-dominated forest, 19 July 2004, $D a$ phne mezereum L., on lower side of leaves, apt., S.V. Buga leg.

\section{Macrosiphum equiseti (Holman, 1961)}

Specimens examined. Vitebsk Prov. Lepel' Distr., BBR, 4 km WSW Domzheritsy Vill., spruce-dominated forest, 20 July 2000, Equisetum sylvaticum L., on stems, apt., S.V. Buga leg.

Macrosiphum euphorbiae (Thomas, 1878)

Specimens examined. Minsk, CBG, rosarium, 12 Aug. 1985, Rosa sp. hort. cv., on apical part of stem, al., S.V. Buga leg. Minsk Prov., Minsk Distr., near Priluki Vill., potato plantation, 7 Sept. 2002, Capsella bursa-pastoris (L.) Med., on inflorescences, al., S.V. Buga leg.

Macrosiphum funestum (Macchiati, 1885)

Specimens examined. Minsk Prov., Starye Dorogi Distr., $3 \mathrm{~km}$ W Falichi RWS, forest glade, 3 Aug. 2000, Rubus idaeus L., on lower side of leaves, al., S.V. Buga leg. 
Macrosiphum gei (Koch, 1855)

Specimens examined. Minsk, CBG, rosarium, 21 June 1990, Rosa sp. hort. cv., on apical part of stem, al., S.V. Buga leg. Minsk Prov., Minsk Distr., near Roshcha RWS, spruce-dominated forest, 3 and 5 June 2009, Geum urbanum L., on flower-bearing stem, apt., N.V. Voronova leg. Vitebsk Prov., Gorodok Distr.: near Zadrach'e (Zadrachje) Vill., 3 July 1996, Anthriscus sylvestris (L.) Hoffm., on inflorescences, apt., S.V. Buga leg.; near Marchenki Vill., 5 June 2000, Anthriscus sylvestris (L.) Hoffm., on inflorescences, apt., S.V. Buga leg.; near Bolotnitsa (Bolotnica) Vill., 6 June 2000, Anthriscus sylvestris (L.) Hoffm., on inflorescences, apt., S.V. Buga leg.; near Rudnia (Rudnya, Rudnja) Vill., 18 June 2008, Anthriscus sylvestris (L.) Hoffm., on inflorescences, apt., al., S.V. Buga leg.

\section{Macrosiphum knautiae Holman, 1972}

Specimens examined. Brest Prov.: Baranovichi Distr., Ivnaja (Ivnaya) RWS, 9 Sept. 2002, Knautia arvensis (L.) Coult., on flower-bearing stem, apt., S.V. Buga leg.; Gantsevichi Distr.: 3.5 km SW Polon' Vill., 14 Sept. 2003, Knautia arvensis (L.) Coult., on flower-bearing stem, apt., S.V. Buga leg.; near Gantsevichi, 13 Sept. 2007, Knautia arvensis (L.) Coult., on flower-bearing stem, apt., S.V. Buga leg.; Ivanovo Distr., near Yukhnevichi RWS, 16 Sept. 2009, Knautia arvensis (L.) Coult., on flower-bearing stem, apt., ovip. fem., S.V. Buga leg.; Ivatsevichi Distr, 1 km N Kholop'ya Vill., 20 Sept. 2004, Knautia arvensis (L.) Coult., on flower-bearing stem, apt., ovip. fem., S.V. Buga leg.; Luninets Distr., near Bostyn' Vill., 16 Sept. 2009, Knautia arvensis (L.) Coult., on flower-bearing stem, apt., S.V. Buga leg.; Malorita Distr.: near Malorita, 29 Aug. 2009, Knautia arvensis (L.) Coult., on flower-bearing stem, apt., S.V. Buga leg.; near Mel'niki Vill., 29 Aug. 2009, Knautia arvensis (L.) Coult., on flower-bearing stem, apt., S.V. Buga leg. Gomel Prov.: Chechersk
Distr., near Bartolomeevskaya Rudnia (Bartolomeevskaya Rudnya, Bartolomeevskaya Rudnja) Vill., 25 Aug. 2005, Knautia arvensis (L.) Coult., on flower-bearing stem, apt., al., S.V. Buga leg.; Dobrush Distr., 1 km NE Dobrush RWS, 22 Aug. 2009, Knautia arvensis (L.) Coult., on flower-bearing stem, apt., S.V. Buga leg.; Oktyabr'skiy Distr.: nearby Moshny RWS, 28 July and 28 Sept. 2009, Knautia arvensis (L.) Coult., on flower-bearing stem, apt., S.V. Buga leg.; near Moshny Vill., 28 Sept. 2009, Knautia arvensis (L.) Coult., on flower-bearing stem, apt., S.V. Buga leg.; Rechitsa Distr., near Kopan' RWS, 31 July 2009, Knautia arvensis (L.) Coult., on flower-bearing stem, apt., S.V. Buga leg. Grodno (Grodna) Prov.: Iv'e (Iv'ye, Iv'ersk) Distr.: NBR, hamlet Naust', 27 Aug. 2002, Knautia arvensis (L.) Coult., on flower-bearing stem, apt., S.V. Buga leg.; near Jachimovshchina RWS, 27 Aug. 2010, Knautia arvensis (L.) Coult., on flower-bearing stem, apt., S.V. Buga leg.; $0.3 \mathrm{~km} \mathrm{~S}$ Jachimovshchina Vill., 27 Aug. 2010, Knautia arvensis (L.) Coult., on flower-bearing stem, apt., S.V. Buga leg.; Slonim Distr.: $3.5 \mathrm{~km}$ WSW Ivnaja (Ivnaya) RWS, 9 Sept. 2002, Knautia arvensis (L.) Coult., on flower-bearing stem, apt., S.V. Buga leg.; $1.5 \mathrm{~km}$ WSW Ivnaja (Ivnaya) RWS, 9 Sept. 2002, Knautia arvensis (L.) Coult., on flower-bearing stem, apt., S.V. Buga leg. Minsk, central part of the city, 28 June 2007, Knautia arvensis (L.) Coult., on apex of stem, apt., N.V. Leshchinskaya leg. Minsk Prov.: Dzerzhinsk Distr.: 0.3 km SES Asino RWS, 27 Aug. 2002, Knautia arvensis (L.) Coult., on flower-bearing stem, apt., S.V. Buga leg.; 27 Sept. 2009, Knautia arvensis (L.) Coult., on flower-bearing stem, male, ovip. fem., S.V. Buga leg.; Kopyl' Distr., near Pechurany Vill., 10 July 2010, Knautia arvensis (L.) Coult., on flower-bearing stem, apt., S.V. Buga leg.; Minsk Distr.: 0.4 km SW Pomyslishche RWS, 9 Okt. 2002, Knautia arvensis (L.) Coult., on stem, ovip. fem., S.V. Buga leg.; near Pomyslishche RWS, 5 July 2004, Knautia arvensis (L.) Coult., on flower-bearing stem, apt., al., S.V. Buga leg.; 
15 July 2005, Knautia arvensis (L.) Coult., on flower-bearing stem, apt., S.V. Buga leg.; Priluki Vill., ruderal vegetation, 8 July 2009, Dipsacus fullonum L., on apical part of stem, apt., al., S.V. Buga leg.; Molodechno Distr., near Udranka Vill., 29 June 2006, Knautia arvensis (L.) Coult., on apex of stem, apt., S.V. Buga leg.; Myadel' Distr., nearby Naroch', 28 June 2007, Knautia arvensis (L.) Coult., on apex of stem, apt., N.V. Leshchinskaya leg.; Nesvizh Distr.: near Lipa RWS, 29 Aug. 2002, Knautia arvensis (L.) Coult., on flower-bearing stem, apt., S.V. Buga leg.; 0.4 km W Lipa RWS, 29 Aug. 2002, Knautia arvensis (L.) Coult., on flower-bearing stem, apt., S.V. Buga leg.; same locality, 14 Sept. 2002, Knautia arvensis (L.) Coult., on flower-bearing stem, ovip. fem., S.V. Buga leg.; Stolbtsy Distr., near Slobodka Vill., 9 July 2010, Knautia arvensis (L.) Coult., on flower-bearing stem, apt., S.V. Buga leg.; Volozhin Distr.: 3 km W Rakov, 26 June 2006, Knautia arvensis (L.) Coult., on apex of stem, apt., S.V. Buga leg.; 0.3 km NE Pral'niki Vill., 27 Aug. 2010, Knautia arvensis (L.) Coult., on flower-bearing stem, apt., S.V. Buga leg. Mogilev Prov.: Belynichi Distr., 1.5 km E Korytnitsa Vill., 23 Aug. 2005, Knautia arvensis (L.) Coult., on flower-bearing stem, apt., S.V. Buga leg.; Klimovichi Distr.: $1 \mathrm{~km} \mathrm{~S}$ vill. Malashkovichi Vill., 24 Aug. 2005, Knautia arvensis (L.) Coult., on flower-bearing stem, apt., S.V. Buga leg.; $4 \mathrm{~km} \mathrm{~N}$ Tserkovishche Vill., 24 Aug. 2005, Knautia arvensis (L.) Coult., on flower-bearing stem, apt., S.V. Buga leg.; Klichev Distr., 5 km NWN Dubno Vill., 15 Aug. 2006, Knautia arvensis (L.) Coult., on flower-bearing stem, apt., al., S.V. Buga leg.; Kostyukovichi Distr., 1 km E Krutoy Rov Vill., 25 Aug. 2005, Knautia arvensis (L.) Coult., on flower-bearing stem, apt., S.V. Buga leg.; Krasnopol'e Distr., 0.3 km S Kholmy Vill., 24 Aug. 2005, Knautia arvensis (L.) Coult., on flower-bearing stem, apt., S.V. Buga leg.; Khotimsk Distr.: 1.5 km NW Markovka Vill., 24 Aug. 2005, Knautia arvensis (L.) Coult., on flower-bearing stem, apt., S.V. Buga leg.; 4 km S Yuze- fovka Vill., 25 Aug. 2005, Knautia arvensis (L.) Coult., on flower-bearing stem, apt., S.V. Buga leg. Vitebsk Prov.: Gorodok Distr.: Bolotnitsa (Bolotnica) Vill., 20 and 22 Aug. 2002, Knautia arvensis (L.) Coult., on flower-bearing stem, apt., S.V. Buga leg.; near Bolotnitsa (Bolotnica) Vill., 22 Aug. 2002, Knautia arvensis (L.) Coult., on flower-bearing stem, apt., S.V. Buga leg.; $1.5 \mathrm{~km}$ WSW Bolotnitsa (Bolotnica) Vill., 22 Aug. 2002, Knautia arvensis (L.) Coult., on flower-bearing stem, apt., S.V. Buga leg.; $0.3 \mathrm{~km}$ W Bolotnitsa (Bolotnica) Vill., 20 and 22 Aug. 2002, Knautia arvensis (L.) Coult., on flower-bearing stem, apt., S.V. Buga leg.; $0.5 \mathrm{~km}$ W Bolotnitsa (Bolotnica) Vill., 6 Aug. 2007, Knautia arvensis (L.) Coult., on flower-bearing stem, apt., S.V. Buga leg.; nearby Laptevka Vill., 19 June 2008, Knautia arvensis (L.) Coult., on apex of stem, apt., al., S.V. Buga leg.; Prudok Vill., 20 Aug. 2002, Knautia arvensis (L.) Coult., on flower-bearing stem, apt., S.V. Buga leg.; $0.5 \mathrm{~km}$ ENE Prudok Vill., 20 Aug. 2002, Knautia arvensis (L.) Coult., on flower-bearing stem, apt., S.V. Buga leg.; nearby Repishche (Repische) Vill., 22 Aug. 2002, Knautia arvensis (L.) Coult., on flower-bearing stem, apt., ovip. fem., S.V. Buga leg.; 5 km WSW Repishche (Repische) Vill., 22 Aug. 2002, Knautia arvensis (L.) Coult., on flower-bearing stem, apt., S.V. Buga leg.; nearby Rudnia (Rudnya, Rudnja) Vill., 19 Aug. 2002, Knautia arvensis (L.) Coult., on flower-bearing stem, apt., S.V. Buga leg.; 3 km E Rudnia (Rudnya, Rudnja) Vill., 19 Aug. 2002, Knautia arvensis (L.) Coult., on flower-bearing stem, apt., S.V. Buga leg.; 3 km NW Rudnia (Rudnya, Rudnja) Vill., 19 Aug. 2002, Knautia arvensis (L.) Coult., on flower-bearing stem, apt., S.V. Buga leg.; $0.3 \mathrm{~km} \mathrm{NW} \mathrm{Rudnia}$ (Rudnya, Rudnja) Vill., 2 Aug. 2007, Knautia arvensis (L.) Coult., on flower-bearing stem, apt., S.V. Buga leg.; $1 \mathrm{~km} \mathrm{NW}$ Zadrach'e (Zadrachje) Vill., 19 Aug. 2002, Knautia arvensis (L.) Coult., on flower-bearing stem, apt., S.V. Buga leg.; 31 July 2007, Knautia arvensis (L.) Coult., on flower-bea- 
ring stem, apt., al., S.V. Buga leg.; Dokshitsy Distr.: 2 km NW Dedino Vill., 7 July 2004, Knautia arvensis (L.) Coult., on flower-bearing stem, apt., S.V. Buga leg.; near Sosnovaya Vill., 3 July 2007, Knautia arvensis (L.) Coult., on flower-bearing stem, apt., S.V. Buga leg.; Miory Distr., $0.5 \mathrm{~km}$ SE Vazho lake, 3 July 2007, Knautia arvensis (L.) Coult., on flower-bearing stem, apt., al., S.V. Buga leg.; Rossony Distr., nearby Krasnopol'e Vill., 1 July 2007, Knautia arvensis (L.) Coult., on apex of stem, apt., S.V. Buga leg.; Ushachi Distr.: 2 km SES Zavydrino Vill., 21 July 2004, Knautia arvensis (L.) Coult., on flower-bearing stem, apt., S.V. Buga leg.; 3 km E Pligovki Vill., 1 July 2007, Knautia arvensis (L.) Coult., on flower-bearing stem, apt., al., S.V. Buga leg.

Macrosiphum melampyri Mordvilko, 1919

Specimens examined. Minsk Prov., Stolbtsy Distr., $1 \mathrm{~km} \mathrm{~N}$ Kolosovo Vill., sprucedominated forest, 13 July 2010, Melampyrum pratense L., on lower side of leaves, apt., S.V. Buga leg.

Macrosiphum nasonovi Mordvilko, 1919

Specimens examined. Minsk Prov.: Minsk Distr., near Kryzhovka Vill., pine forest, 16 July 2000, Vaccinium uliginosum L., al., S.V. Buga leg.; Volozhin Distr., NBR, near hamlet Pechishche, raised bog, 25 July 1987, Vaccinium uliginosum L., on apical part of stem, apt., S.V. Buga leg.

Published information. Gomel Prov.: Petrikov Distr., Petrikov (Mordvilko, 1930); Svetlogorsk Distr., Yakimova Sloboda Vill. (Mordvilko, 1930).

\section{Macrosiphum rosae (Linnaeus, 1758)}

Specimens examined. Brest Prov., Zhabinka Distr., vill Bol'shye Yakovchitsy, green stands, 17 Oct. 2000, Rosa rugosa Thunb., on lower side of leaves, gyn., S.V. Buga leg. Grodno (Grodna) Prov., Lida Distr., $1.3 \mathrm{~km}$ NNE Neman RWS, dry meadow, 31 Aug.
2000, Rosa sp., on lower side of leaves, apt., al., S.V. Buga leg. Minsk: CBG, rosarium, 21 June 1990, Rosa sp. hort. cv., on apical part of stem, apt., al., S.V. Buga leg.; Central part of the city, green stands, 4 Nov. 2000, Rosa rugosa Thunb., on lower side of leaves, gyn., S.V. Buga leg.; CBG, roadside, ruderal vegetation, 15 June 2006, Valeriana officinalis L., on steam near inflorescences, al., N.V. Leshchinskaya leg. Minsk Prov.: Borisov Distr., Borisov, gardens, 2 July 1987, Rosa sp. hort. cv., on apical part of stem, apt., al., S.V. Buga leg.; Cherven' Distr., 2 km E Ozerny Vill., bushes, 6 July 2000, Rosa sp., on apical part of stem, apt., S.V. Buga leg.; Minsk Distr., Priluki Vill., 4 June 1985, Rosa alba L., on apical part of stem, apt., al., S.V. Buga leg.; Stolbtsy Distr., NBR, near Kletishche Vill., floodplain of Usa river, bushes, 14 July 2010, Rosa glauca Pourr., on apical part of stem, apt., S.V. Buga leg.; Volozhin Distr., $0.3 \mathrm{~km}$ E Yurzhishki Vill., green stands, 27 Aug. 2010, Rosa sp., on apical part of stem, apt., S.V. Buga leg. Mogilev Prov., Gorki Distr., Zubry RWS, strip-shaped forest patch, 10 Oct. 2000, Rosa rugosa Thunb., on lower side of leaves, ovip. fem., S.V. Buga leg. Vitebsk Prov.: Gorodok Distr:: Obol' (Obolj) Vill., green stands, 19 June 2008, Rosa sp., R. Rakauskas leg.; Laptevka, 19 June 2008, Knautia arvensis (L.) Coult., on apical part of stem, apt., S.V. Buga leg.; Polotsk Distr., Polotsk, green stands, 2 July 2007, Rosa sp., on lower side of leaves, apt., S.V. Buga leg.; Postavy Distr., Postavy, green stands, 20 July 2010, Rosa sp. hort. cv., on apical part of stem, apt., S.V. Buga leg.

\section{Megoura viciae Buckton, 1876}

Specimens examined. Grodno (Grodna) Prov., Iv'e (Iv'ye, Iv'evsk) Distr.: near Yahimovshchina RWS, dry meadow, 27 Aug. 2010, Lathyrus pratensis L., on apical part of stem, apt., S.V. Buga leg.; near Kryzhniki Vill., dry meadow, 27 Aug. 2010, Lathyrus pratensis L., on apical part of stem, apt., S.V. Buga leg. Minsk Prov.: Minsk Distr.: near Shchemyslitsa Vill., young lime forest, 31 
July 1986, Lathyrus pratensis L., on apical part of stem, apt., S.V. Buga leg.; $0.8 \mathrm{~km}$ SW Pomyslishche RWS, meadow, 24 Aug. 1998, Vicia cracca L., on apical part of stem, apt., al., S.V. Buga leg.; Nesvizh Distr., 0.2 km N Lipa RWS, pine forest, 6 Sept. 2000, Lathyrus sp., on stem, apt., S.V. Buga leg.; Vileyka Distr., $2 \mathrm{~km}$ NNE Khotenchitsy Vill., mixed forest, 26 May 2005, Lathyrus pratensis L., on apical part of stem, al., S.V. Buga leg. Vitebsk Prov., Ushachi Distr., near Urbanoe Vill., dry meadow, 21 July 2004, Lathyrus pratensis L., on apical part of stem, apt., al., S.V. Buga leg.

\section{Metopeurum fuscoviride Stroyan, 1950}

Specimens examined. Grodno (Grodna) Prov., Smorgon' Distr., Soly Vill., ruderal vegetation, 24 June 2006, Tanacetum vulgare L., apices of stem, apt., N.V. Leshchinskaya leg. Mogilev Prov.: Krasnopol'e Distr., 1 km NW Vydrenka Vill., dry meadow, 25 Aug. 2005, Tanacetum vulgare L., on inflorescence, apt., S.V. Buga leg.; Khotimsk Distr., 4 km S Yuzefovka Vill., oak-dominated forest with species-rich grass cover, 25 Aug. 2005, Tanacetum vulgare L., on inflorescence, apt., S.V. Buga leg. Vitebsk Prov.: Gorodok Distr., Zadrach'e (Zadrachje) Vill., ruderal vegetation, 5 Aug. 2007, Tanacetum vulgare L., apt., S.V. Buga leg.; Rossony Distr., Klyastitsy Vill., ruderal vegetation, 19 Aug. 2005, Tanacetum vulgare L., on inflorescence, apt., S.V. Buga leg.

Metopolophium dirhodum (Walker, 1849)

Specimens examined. Brest Prov., Zhabinka Distr., Bol'shye Yakovchitsy Vill., green stands, 17 Oct. 2000, Rosa rugosa Thunb., on lower side of leaves, gyn., S.V. Buga leg. Gomel Prov.: Oktyabr'skiy Distr., near Moshny RWS, green stands, 28 Sept. 2009, Rosa rugosa Thunb., on lower side of leaves, gyn., males, S.V. Buga leg.; Rogachev Distr., 1.4 km S Vitlin Vill., strip-shaped forest patch, 20 Oct. 2000, Rosa rugosa Thunb., on lower side of leaves, gyn., males, ovip. fem., S.V. Buga leg. Grodno (Grodna) Prov., Zel'va Distr.: $1.5 \mathrm{~km} \mathrm{NNE}$ Rostevichi Vill., pine forest, 14 Oct. 2000, Rosa glauca Pourr., on lower side of leaves, gyn., S.V. Buga leg.; same locality, green stands, 1 Nov. 2000, Rosa sp. hort. cv., on lower side of leaves, gyn., males, ovip. fem., S.V. Buga leg.; Pletyanichi RWS, strip-shaped forest patch, 14 Oct. 2000, Rosa rugosa Thunb., on lower side of leaves, gyn., S.V. Buga leg. Minsk: CBG, arboretum, 29 Sept. 1987, Rosa pisocarpa A. Grey, on lower side of leaves, gyn., S.V. Buga leg.; same locality, 1 Oct. 1987, Rosa boissieri Crep., on lower side of leaves, gyn., S.V. Buga leg.; Central part of the city, green stands, 4 Nov. 2000 , Rosa rugosa Thunb., on lower side of leaves, gyn., ovip. fem., S.V. Buga leg.; green stands, 7 Nov. 2000, Rosa rugosa Thunb., on lower side of leaves, gyn., ovip. fem., S.V. Buga leg. Minsk Prov., Minsk Distr., 0.8 km SW Pomyslishche RWS, 7 Nov. 2000, Rosa rugosa Thunb., on lower side of leaves, gyn., males, ovip. fem., S.V. Buga leg.; Priluki Vill., green stands, 27 Nov. 2003, Rosa sp. hort. cv., on lower side of leaves, males, ovip. fem., S.V. Buga leg. Mogilev Prov.: Bobruysk Distr., $1.5 \mathrm{~km}$ E Danilov Most Vill., strip-shaped forest patch, 20 Oct. 2000, Rosa glauca Pourr., on lower side of leaves, gyn., S.V. Buga leg.; Gorki Distr., Zubry RWS, strip-shaped forest patch, 10 Oct. 2000, Rosa rugosa Thunb., on lower side of leaves, gyn., males, ovip. fem., S.V. Buga leg.; Kostyukovichi Distr., 0.4 km NE Belynkovichi Vill., floodplain of river Besed', dry meadow, 6 Oct. 2000, Rosa glauca Pourr., on lower side of leaves, gyn., S.V. Buga leg.; Krichev Distr., Krichev, green stands, 10 Oct. 2000, Rosa glauca Pourr., on lower side of leaves, gyn., males, ovip. fem., S.V. Buga leg. Vitebsk Prov.: Postavy Distr., 0.4 km E Myadel'ka Vill., green stands, 29 Sept. 2000, Rosa rugosa Thunb., on lower side of leaves, gyn., S.V. Buga leg.; Senno Distr., $2.3 \mathrm{~km}$ E Lipno Vill., green stands, 27 Sept. 2000, Rosa rugosa Thunb., on lower side of leaves, gyn., S.V. Buga leg. 
Microlophium carnosum (Buckton, 1876)

Specimens examined. Brest Prov., $\mathrm{Ka}$ menets Distr., BPF, near Kamenyuki Vill., black alder forest with nettle cover, 13 Oct. 1999, Urtica dioica L., on lower side of leaves, ovip. fem., S.V. Buga leg. Vitebsk Prov., Gorodok Distr., Zadrach'e (Zadrachje) Vill., 13 July 2000, Urtica dioica L., on lower side of leaves, apt., S.V. Buga leg.

Published information. Vitebsk Prov., BBR (as Microlophium evansi Theob.) (Buga, 1989).

\section{Microsiphum millefolii Wahlgren, 1940}

Specimens examined. Vitebsk Prov., Lepel' Distr., BBR, near Domzheritsy Vill., dry meadow, 15 Aug. 1986, Achillea millefolium L. s.l., on apical part of stem, apt., S.V. Buga leg.

Microsiphum woronieckae Judenko, 1931

Specimens examined. Vitebsk Prov., Gorodok Distr., Rudnia (Rudnya, Rudnja) Vill., wasteland, 18 June 2008, Artemisia vulgaris L., on shoots, apt., R. Rakauskas leg.

\section{Myzaphis bucktoni (Jacob, 1946)}

Specimens examined. Minsk, CBG, arboretum, 14 June 1995, Rosa sp., in leaf gall, apt., S.V. Buga leg.

\section{Myzaphis rosarum (Kaltenbach, 1843)}

Specimens examined. Minsk: CBG, arboretum, 18 July 1986, Pentaphylloides $\times$ friedrichsenii (Spath ex C.K.Schneid.) Soják, on lower side of leaves, apt., S.V. Buga leg.; arboretum, 18 July 1986, Pentaphylloides fruticosa (L.) O. Schwarz, on lower side of leaves, apt., S.V. Buga leg.; arboretum, 29 July 1987, Rosa sp., in leaf gall, apt., S.V. Buga leg. Minsk Prov., Minsk Distr., Priluki Vill., green stands, 7 July 2010, Rosa sp., in leaf gall, apt., S.V. Buga leg.
Myzus cerasi cerasi (Fabricius, 1775)

Specimens examined. Brest Prov., Kamenets Distr., BPF, Kamenyuki Vill., green stands, 13 Oct. 1999, Cerasus vulgaris L. hort. cv., on lower side of leaves, ovip. fem., S.V. Buga leg. Grodno (Grodna) Prov., Grodno (Grodna) Distr., Grodno (Grodna), green stands, 26 May 2004, Cerasus vulgaris L. hort. cv., on lower side of leaves, apt., D.L. Petrov leg. Minsk, orchard, 16 July 2009, Cerasus vulgaris L. hort. cv., on lower side of leaves, apt., al., S.V. Buga leg. Minsk Prov.: Borisov Distr., Borisov, orchard, 2 July 1987, Cerasus vulgaris L. hort. cv., on lower side of leaves, apt., al., S.V. Buga leg.; Minsk Distr., Priluki Vill., orchard, 1 July 1983, Cerasus vulgaris L. hort. cv., on lower side of leaves, apt., S.V. Buga leg.; Myadel' Distr., Antonisberg Vill., orchard, 2 July 2009, Cerasus vulgaris L. hort. cv., on apical part of stem, apt., L.A. Serbina.; Stolbtsy Distr., Stolbtsy, orchard, 14 July 2010, Cerasus vulgaris L. hort. cv., on lower side of leaves, apt., S.V. Buga leg. Vitebsk Prov.: Braslav Distr., Gayleshi Vill., orchard, 3 July 2007, Cerasus vulgaris L. hort. cv., on lower side of leaves, apt., al., S.V. Buga leg.; Gorodok Distr.: Gorodok, green stands, 17 June 2008, Cerasus vulgaris L. hort. cv., R. Rakauskas leg.; Zadrach'e (Zadrachje) Vill., 18 June 2008, Cerasus vulgaris L. hort. cv., apt., R. Rakauskas leg.; Miory Distr., Perebrod'e Vill., orchard, 3 July 2007, Cerasus vulgaris L. hort. cv., on apical part of stem, apt., al., S.V. Buga leg.; Postavy Distr., Postavy, orchard, 20 July 2010, Cerasus vulgaris L. hort. cv., on lower side of leaves, apt., S.V. Buga leg.

Published information. Vitebsk Prov., Sharkoushchina (Szarkoreszczyzna) Distr., Bazylpol, 3 Sept. 1924, Prunus insititia L. (as Aphis cerasi) (Fiedorowiczówna, 1930).

Myzus cerasi pruniavium Börner, 1926

Specimens examined. Brest Prov., Pinsk Distr., Pinsk, orchard, 7 June 1990, Cerasus avium (L.) Moench. hort. cv., on lower side of leaves, apt., S.V. Buga leg. Minsk Prov., 
Nesvizh Distr., Nesvizh, green stands, 10 July 2010, Cerasus avium (L.) Moench. hort. cv., on apical part of stem, apt., S.V. Buga leg.

Myzus ligustri (Mosley, 1841)

Specimens examined. Minsk Prov., Minsk Distr., Pomyslishche RWS, green stands, 4 July 2000, Ligustrum vulgare L., on apical part of stem, apt., S.V. Buga leg.

Myzus lythri (Schrank, 1801)

Specimens examined. Minsk: green stands, 23 and 26 June 2000, Prunus mahaleb L., on apical part of stem, apt., al., S.V. Buga leg.; green stands, 6 July 2000, Prunus mahaleb L., on apical part of stem, apt., S.V. Buga leg.

\section{Myzus padellus}

Hille Ris Lambers et Rogerson, 1946

Specimens examined. Minsk, CBG, arboretum, 10 July 1986, Padus racemosa (Lam.) Gilib., on lower side of leaves, apt., S.V. Buga leg.

\section{Myzus (Galiobium) langei}

(Börner, 1933)

Specimens examined. Minsk Prov., Molodechno Distr., near Udranka Vill., dry meadow, 22 May 2008, Galium mollugo L., on stem, fund., apt., S.V. Buga leg.

\section{Myzus (Nectarosiphon) certus}

(Walker, 1849)

Specimens examined. Brest Prov., Bereza Distr., near Bereza, dry mixed forest, 10 June 2008, Dianthus arenarius L., on apical part of stem, apt., al., N.V. Leshchinskaya leg.

\section{Myzus (Nectarosiphon) persicae} (Sulzer, 1776)

Specimens examined. Minsk: CBG, arboretum, 4 June 1985, Hydrangea cinerea Small, on apical part of stem, al., S.V. Buga leg.; rosarium, 9 Aug. 1985, Rosa sp. hort. cv., on lower side of leaves, apt., S.V. Buga leg.; arboretum, 29 July 1987, Artemisia abrotanum L., on lower side of leaves, apt., S.V. Buga leg.

\section{Myzus (Sciamyzus) ascalonicus}

Doncaster, 1946

Specimens examined. Minsk, CBG, greenhouse, 21 June 1990, Freesia refracta (Jacq.) Ecklon. cv., on apical part of stem, apt., S.V. Buga leg.

\section{Nasonovia pilosellae (Börner, 1933)}

Specimens examined. Brest Prov., Bereza Distr., near Bereza, dry meadow, 10 June 2008, Pilosella officinarum F.W. Schultz et Sch.Bip., on apical part of stem, apt., N.V. Leshchinskaya leg.

\section{Nasonovia ribisnigri (Mosley, 1841)}

Specimens examined. Brest Prov., Kamenets Distr: BPF, near Kamenyuki Vill., mixed forest, 13 Oct. 1999, Ribes lucidum Kit., on lower side of leaves, ovip. fem., S.V. Buga leg.; 0.2 km N Kamenyuki Vill., alder forest with nettle cover, 14 Oct. 1999, Ribes lucidum Kit., on lower side of leaves, gyn., S.V. Buga leg.; BPF, near Kamenyuki Vill., spruce-dominated forest, 22 Sept. 2000, Ribes lucidum Kit., on lower side of leaves, ovip. fem., A.A. Ponomarev leg. Vitebsk Prov., Gorodok Distr., near Studenka (Studjenka) Vill., dry meadow, 19 June 2008, Pilosella officinarum F.W. Schultz et Sch.Bip., apt., S.V. Buga leg.

Neoamphorophora ledi (Wahlgren, 1938)

Specimens examined. Mogilev Prov., Bykhov Distr., Gribovetskoe forestry, raised bog, 24 May 1991, Ledum palustre L., apt., S.V. Buga leg.

\section{Neomyzus circumflexus (Buckton, 1876)}

Specimens examined. Minsk: CBG, rosarium, 12 Aug. 1985, Rosa sp. hort. cv., on 
apical part of stem, apt., al., S.V. Buga leg.; same locality and date, Salvia sp. hort. cv., on lower side of leaves, apt., al., S.V. Buga leg.

\section{Ovatus crataegarius (Walker, 1850)}

Specimens examined. Minsk, green stands, 20 Sept. 1999, Crataegus sp., on lower side of leaves, males, ovip. fem., S.V. Buga leg.

\section{Paramyzus heraclei Börner, 1933}

Specimens examined. Minsk Prov., Volozhin Distr., $0.3 \mathrm{~km}$ NE Pral'niki Vill., mixed forest, 27 Aug. 2010, Heracleum sibiricum L., on lower side of leaves, apt., S.V. Buga leg.

\section{Phorodon humuli (Schrank, 1801)}

Specimens examined. Brest Prov.: $\mathrm{Ka}$ menets Distr., BPF, Kamenyuki Vill., green stands, 14 Oct. 1999, Prunus divaricata Ledeb. hort. cv., on lower side of leaves, gyn., ovip. fem., S.V. Buga leg.; Lyakhovichi Distr., $0.5 \mathrm{~km}$ SE Zadvor'e Vill., bushes, 8 Aug. 2000, Humulus lupulus L., on lower side of leaves, apt., S.V. Buga leg.; Malorita Distr., Malorita, orchard, 29 Aug. 2009, Humulus lupulus L., on lower side of leaves, apt., S.V. Buga leg.; Stolin Distr., 3 km ESE Korob'e Vill., floodplain oak-dominated forest, 3 Oct. 2000, Humulus lupulus L., on lower side of leaves, gyn., S.V. Buga leg.; Zhabinka Distr., Bol'shye Yakovchitsy Vill., green stands, 17 Oct. 2000, Prunus domestica L. hort. cv., on lower side of leaves, ovip. fem., S.V. Buga leg. Gomel Prov.: Rechitsa Distr., Rechitsa, orchard, 31 July 2009, Humulus lupulus L., on lower side of leaves, apt., S.V. Buga leg.; Zhitkovichi Distr.: $10 \mathrm{~km} \mathrm{~S}$ Khvoensk Vill., swamped forest, 11 July 1999, Humulus lupulus L., on lower side of leaves, apt., S.V. Buga leg.; 4 km NW Khvoensk Vill., floodplain of Pripyat river, forest with dense undergrowth, 12 July 1999, Humulus lupulus L., on lower side of leaves, apt., S.V. Buga leg. Grodno
(Grodna) Prov:: Iv'e (Iv'ye, Iv'evsk) Distr., $0.75 \mathrm{~km}$ WSW Gav'ya RWS, black alder wood in a brook valley, 9 Sept. 2000, Huтulus lupulus L., on lower side of leaves, apt., S.V. Buga leg.; Smorgon' Distr., 0.4 km ENE Degisi Vill., alder forest with species-rich grass cover, 2 Sept. 2000, Humulus lupulus L., on lower side of leaves, apt., S.V. Buga leg. Minsk, Loshitsa, 30 June 1924, Prunus sp., apt., E. Yatsenkovsky leg. Minsk Prov.: Borisov Distr., Borisov, Berezina river valley, bushes, 2 July 1987, Humulus lupulus L., on lower side of leaves, apt., S.V. Buga leg.; Cherven' Distr., 2 km E Ozerny Vill., bushes, 6 July 2000, Humulus lupulus L., on lower side of leaves, apt., S.V. Buga leg.; Dzerzhinsk Distr., near Mezinovka RWS, green stands, 13 June 2000, Prunus divaricata Ledeb., on lower side of leaves, al., S.V. Buga leg.; Minsk Distr.: near Priluki Vill., floodplain forest, 23 Aug. 1985, Humulus lupulus L., on lower side of leaves, apt., S.V. Buga leg.; near Priluki Vill., floodplain forest, 4 Oct. 1987, Humulus lupulus L., on lower side of leaves, ovip. fem., S.V. Buga leg.; Priluki Vill., orchard, 13 June 1999, Prunus divaricata Ledeb. hort. cv., on lower side of leaves, apt., S.V. Buga leg.; same locality and date, 24 July 2009, Prunus divaricata Ledeb. hort. cv., on lower side of leaves, al., S.V. Buga leg.; Nesvizh Distr., Nesvizh, green stands, 10 July 2010, Prunus divaricata Ledeb. hort. cv., on lower side of leaves, apt., S.V. Buga leg. Mogilev Prov., Kostyukovichi Distr., $0.4 \mathrm{~km}$ NE Belynkovichi Vill., floodplain of Besed' river, willow growth in floodplain, 6 Oct. 2000, Humulus lupulus L., on lower side of leaves, male, S.V. Buga leg. Vitebsk Prov.: Gorodok Distr., Zadrach'e (Zadrachje) Vill., 18 June 2008, Prunus domestica L. hort. cv., R. Rakauskas leg.; Ushachi Distr., 2 $\mathrm{km}$ SSE Zavydrino Vill., spruce-dominated forest, 21 July 2004, Humulus lupulus L., on lower side of leaves, apt., S.V. Buga leg.

Published information. Vitebsk Prov., Sharkoushchina (Szarkoreszczyzna) Distr., Bazylpol, 3 Sept. 1924, Prunus insititia L. (Fiedorowiczówna, 1930). 


\section{Pleotrichophorus deviatus}

F.P. Müller, 1972

Specimens examined. Minsk Prov., Starye Dorogi Distr., near Paseka RWS, pine forest, 7 Oct. 2005, Pilosella officinarum F.W. Schultz et Sch.Bip., on upper and lower side of leaves, ovip. fem., S.V. Buga leg.

\section{Rhopalomyzus (Judenkoa) lonicerae (Siebold, 1839)}

Specimens examined. Grodno (Grodna) Prov., Smorgon' Distr., 3.3 km NNE Soly RWS, pine forest, 2 Sept. 2000, Lonicera xylosteum L., on lower side of leaves, gyn., S.V. Buga leg. Minsk Prov.: Minsk Distr., $1 \mathrm{~km}$ NE Priluki Vill., mixed forest, 1 Oct. 1987, Lonicera xylosteum L., on lower side of leaves, ovip. fem., S.V. Buga leg.; Molodechno Distr., near Udranka Vill., 24 July 2000 , Phalaris arundinacea L., on lower side of leaves, apt., I.P. Moskvina leg.; Nesvizh Distr., $0.4 \mathrm{~km} \mathrm{~W} \mathrm{Lipa} \mathrm{RWS,} \mathrm{alder} \mathrm{forest}$ with species-rich grass cover, 6 Sept. 2000, Lonicera xylosteum L., on lower side of leaves, gyn., S.V. Buga leg. Mogilev Prov., Gorki Distr., 0.8 km N Kukshinovo Vill., spruce-dominated forest, 10 Oct. 2000, Lonicera xylosteum L., on lower side of leaves, gyn., S.V. Buga leg. Vitebsk Prov., Postavy Distr., 0.4 km E Myadel'ka Vill., spruce-dominated forest, 29 Sept. 2000, Lonicera xylosteum L., on lower side of leaves, gyn., S.V. Buga leg.

Semiaphis anthrisci (Kaltenbach, 1843)

Specimens examined. Minsk Prov., Minsk Distr., Sosny forestry, ruderal vegetation, 18 Sept. 2007, Torilis japonica (Houtt.) DC., apt., ovip. fem., N.V. Leshchinskaya leg.

Semiaphis pimpinellae (Kaltenbach, 1843)

Specimens examined. Grodno (Grodna) Prov., Korelichi Distr., near Korelichi, dry meadow, 4 Aug. 2007, Pimpinella saxifraga L., on root collar, apt., N.V. Leshchinskaya leg.
Published information. Grodno (Grodna) Prov., Oshmyany (Oshmjany) Distr., Oshmyany (Oshmjany), 1 Sept. 1932, Pimpinella saxifraga L. (as Aphis anthrisci Kalt.) (Perepeczko-Baumanowa, 1934) (?Semiaphis pimpinellae (Kalt.)).

\section{Sitobion avenae (Fabricius, 1775)}

Specimens examined. Gomel Prov., Mozyr' Distr.: Mozyr', 9 Aug. 1929, Avena sativa L., apt., al., A.K. Mordvilko leg.; Skrygalovo, 13 Aug. 1929, Avena sativa L., apt., A.K. Mordvilko leg. Minsk Prov.: Minsk Distr.: $1 \mathrm{~km}$ S Pomyslishche RWS, grain crop field, 25 June 2000, Secale cereale L. cv. and Triticum sp. cv., on ears, apt., al., S.V. Buga leg.; near Kryzhovka RWS, dry pine forest, 5 July 2006, Calamagrostis arundinacea (L.) Roth, on panicle, apt., N.V. Leshchinskaya leg.; Pukhovichi Distr., $1 \mathrm{~km}$ SW Dem'yanovka Vill., pine forest, 16 June 2000, Dactylis glomerata L., on panicle, apt., al., S.V. Buga leg.; Starye Dorogi Distr.: Yazyl', 1910, Echinochloa crus-galli (L.) Beauv., al., A.K. Mordvilko leg.; same locality, 14 June 1910, Secale cereale L., apt., al., A.K. Mordvilko leg.; same locality and date, Hordeum vulgare L., apt., A.K. Mordvilko leg. Vitebsk Prov., Gorodok Distr., near Zadrach'e (Zadrachje) Vill., 2 June 2000, Secale cereale L. cv., al., S.V. Buga leg.

Published information. Gomel Prov., Mozyr' Distr., Skrygalovo (Mordvilko, 1930).

\section{Sitobion fragariae (Walker, 1848)}

Specimens examined. Brest Prov., Kamenets Distr: BPF, near Kamenyuki Vill., edge of mixed forest, 13 Oct. 1999, Rubus idaeus L., on lower side of leaves, gyn., S.V. Buga leg.; same locality, but alder forest with whortleberry cover, 13 Oct. 1999, $R u$ bus nessensis W.Hall, on lower side of leaves, ovip. fem., S.V. Buga leg.; 0.2 km N Kamenyuki Vill., alder forest with nettle cover, 14 Oct. 1999, Rubus caesius L., on lower side of leaves, gyn., S.V. Buga leg. 
Sitobion paludum F.P. Müller, 1982

Specimens examined. Brest Prov., $\mathrm{Ka}$ menets Distr., BPF, near Kamenyuki Vill., local raised bog, 30 Apr. 1989, Vaccinium uliginosum L., on lower side of leaves, em., S.V. Buga leg.

\section{Titanosiphon artemisiae (Koch, 1855)}

Specimens examined. Grodno (Grodna) Prov., Korelichi Distr., near Korelichi, dry meadow, 4 Aug. 2008, Artemisia campestris L., on lower side of leaves, apt., N.V. Leshchinskaya leg. Minsk Prov., Starye Dorogi Distr., near Paseka RWS, pine forest, 7 Oct. 2005, Artemisia vulgaris L., on lower side of leaves, apt., S.V. Buga leg.

Titanosiphon minkiewiczi Judenko, 1931

Specimens examined. Vitebsk Prov., Gorodok Distr., Zadrach'e (Zadrachje) Vill., 31 July 2007, Artemisia vulgaris L., on lower side of leaves, apt., S.V. Buga leg.

\section{Trichosiphonaphis (Xenomysus) corticis (Aizenberg, 1935)}

Specimens examined. Grodno (Grodna) Prov., Smorgon' Distr:: 2 km SW Rachuny Vill., mixed forest, 2 Sept. 2000, Lonicera xylosteum L., lignified and non-lignified shoots, apt., S.V. Buga leg.; same locality, 4 Oct. 2005, Lonicera xylosteum L., on 2-4year shoots, ovip. fem., S.V. Buga leg. Mogilev Prov., Gorki Distr., Zubry RWS, green stands, 20 June 2008, Lonicera sp., lignified and non-lignified shoots, al., S.V. Buga leg.

\section{Tubaphis ranunculina (Walker, 1852)}

Specimens examined. Gomel Prov., Oktyabr'skiy Distr., near Moshny RWS, mixed forest, 28 Sept. 2009, Ranunculus repens L., on lower side of leaves, apt., S.V. Buga leg. Grodno (Grodna) Prov., Smorgon' Distr., 2 km SW Rachuny Vill., ruderal vegetation, 4 Oct. 2005, Ranunculus repens L., on lower side of leaves, ovip. fem., S.V.
Buga leg. Minsk, CBG, ruderal vegetation, 16 Sept. 1997, Ranunculus repens L., on lower side of leaves, apt., S.V. Buga leg. Mogilev Prov.: Chausy Distr., 3 km S Maly Gryazivets Vill., spruce-dominated forest, 24 Aug. 2005, Ranunculus repens L., on lower side of leaves, al., S.V. Buga leg.; Gorki Distr., 0.8 km N Kukshinovo Vill., black alder wood in brook valley, 10 Oct. $2000, R a-$ nunculus repens $\mathrm{L}$., on lower side of leaves, ovip. fem., S.V. Buga leg.; Klimovichi Distr., $4 \mathrm{~km} \mathrm{~N}$ Tserkovishche Vill., oak-dominated forest with species-rich grass cover, 24 Aug. 2005, Ranunculus repens L., on lower side of leaves, al., S.V. Buga leg.; Kostyukovichi Distr., 1.5 km W Ruchey Vill., oak-dominated forest, 25 Aug. 2005, Ranunculus repens L., on lower side of leaves, al., S.V. Buga leg.; Krasnopol'e Distr., 0.3 km S Kholmy Vill., alder forest with species-rich grass cover, 24 Aug. 2005, Ranunculus repens L., on lower side of leaves, al., S.V. Buga leg.; Krichev Distr., 0.6 km SE Prygovka Vill., oak-dominated forest in a brook valley, 24 Aug. 2005, Ranunculus repens L., on lower side of leaves, al., S.V. Buga leg. Vitebsk Prov., Senno Distr., 2.4 km E Lipno Vill., oak-dominated forest with bracken fern cover, 27 Sept. 2000, Ranunculus repens L., on lower side of leaves, male, ovip. fem., S.V. Buga leg.

\section{Uroleucon achilleae (Koch, 1855)}

Specimens examined. Minsk: wasteland, 5 Oct. 1997, Achillea millefolium L. s.l., on lower side of leaves, apt., S.V. Buga leg.; ruderal vegetation, 15 Oct. 1997, Achillea millefolium L. s.l., on lower side of leaves, males, S.V. Buga leg.

\section{Uroleucon cichorii cichorii (Koch, 1855)}

Specimens examined. Brest Prov., Lyakhovichi Distr., Lyakhovichi RWS, dry meadow, 8 Aug. 2000, Cichorium intybus L., on apical part of stem, apt., S.V. Buga leg. Minsk Prov., Minsk Distr., Priluki Vill., dry meadow, 24 Aug. 1986, Cichorium intybus L., on apical part of stem, apt., S.V. Buga leg. 


\section{Uroleucon cichorii leontodontis}

(Hille Ris Lambers, 1939)

Specimens examined. Minsk, CBG, landscape park, 21 Aug. 1985, Leontodon autumnalis L., on inflorescences, apt., S.V. Buga leg. Vitebsk Prov., Lepel' Distr., BBR, near hamlet Savskiy Bor, pine forest, 25 July 1986, Leontodon autumnalis L., on apical part of stem, apt., S.V. Buga leg.

\section{Uroleucon cirsii (Linnaeus, 1758)}

Specimens examined. Minsk Prov., Minsk Distr., Priluki Vill., ruderal vegetation, 23 Aug. 1995, Cirsium arvense (L.) Scop., on apical part of stem, apt., S.V. Buga leg.

\section{Uroleucon hypochoeridis}

(Fabricius, 1779)

Specimens examined. Vitebsk Prov., Tolochin Distr., $3 \mathrm{~km}$ N Staraya Budovka Vill., 21 June 2008, Crepis biennis L., on stem near inflorescences, apt., al., S.V. Buga leg.

\section{Uroleucon murale (Buckton, 1876)}

Specimens examined. Grodno (Grodna) Prov., Smorgon' Distr., 2 km SW Rachuny Vill., forest road, 4 Oct. 2005, Mycelis muralis (L.) Dumort, on inflorescences, ovip. fem., S.V. Buga leg. Minsk Prov., Minsk Distr., near Roshcha RWS, spruce-dominated forest, 9 Sept. 2005, Mycelis muralis (L.) Dumort, on flower-bearing stem, apt., S.V. Buga leg. Mogilev Prov., Kostyukovichi Distr., 1.5 km W Ruchey Vill., oak-dominated forest, 25 Aug. 2005, Mycelis muralis (L.) Dumort, on flower-bearing stem, apt., S.V. Buga leg. Vitebsk Prov., Lepel' Distr., BBR, 3 km SW Perekhodtsy Vill., spruce-dominated forest, 8 July 2004, Mycelis muralis (L.) Dumort, on flower-bearing stem, apt., S.V. Buga leg.

\section{Uroleucon obscurum (Koch, 1855)}

Specimens examined. Gomel Prov., Zhitkovichi Distr., 2 km NNE Novolenin Vill., pine forest, 15 Sept. 2004, Hieracium umbellatum L., bases on inflorescences, male, ovip. fem., S.V. Buga leg.

\section{Uroleucon picridis (Fabricius, 1775)}

Specimens examined. Brest Prov., Gantsevichi Distr., 3.5 km SW Polon' Vill., meadow, 14 Sept. 2003, Picris hieracioides L., on flower-bearing stem, apt., S.V. Buga leg. Minsk Prov., Molodechno Distr., near Udranka Vill., dry meadow, 17 July 1995, Picris hieracioides L., on flower-bearing stem, apt., S.V. Buga leg.

\section{Uroleucon pilosellae (Börner, 1933)}

Specimens examined. Brest Prov., Bereza Distr., near Bereza, dry meadow, 10 June 2008, Pilosella officinarum F.W. Schultz et Sch.Bip., on apical part of stem, apt., al., N.V. Leshchinskaya leg.

\section{Uroleucon pseudobscurum (Hille Ris Lambers, 1967)}

Specimens examined. Brest Prov., Bereza Distr., nearby Bereza, dry meadow, 10 June 2008, Hieracium sp., on apical part of stem, apt., N.V. Leshchinskaya leg. Mogilev Prov., Osipovichi Distr., near Verkhi RWS, mixed forest, 1 July 2008, Hieracium umbellatum L., on stem, apt., N.V. Leshchinskaya leg.

Uroleucon pulicariae (Hille Ris Lambers, 1939)

Specimens examined. Gomel Prov., Svetlogorsk Distr., Yakimova Sloboda Vill., 2 Aug. 1929, Inula sp., on stem, apt., A.K. Mordvilko leg.

\section{Uroleucon sonchi (Linnaeus, 1767)}

Specimens examined. Minsk Prov., Minsk Distr., Priluki Vill., ruderal vegetation, 25 Aug. 1995, Sonchus arvensis L., on inflorescences, apt., S.V. Buga leg. Vitebsk Prov., Gorodok Distr.: Zadrach'e (Zadrachje) Vill., 4 Aug. 2007, Sonchus arvensis L., on 
flower-bearing stem, apt., S.V. Buga leg.; Prudok Vill., 6 Aug. 2007, Sonchus oleraceus L., on flower-bearing stem, apt., S.V. Buga leg.

\section{Uroleucon tanaceti (Linnaeus, 1758)}

Specimens examined. Minsk Prov., Minsk Distr., near Roshcha RWS, dry meadow, 8 July 2007, Tanacetum vulgare L., on lower side of leaves, apt., N.V. Leshchinskaya leg.

\section{Uroleucon tussilaginis (Walker, 1850)}

Specimens examined. Gomel Prov., Svetlogorsk Distr., Yakimova Sloboda Vill., 23 July 1929, apt., A.K. Mordvilko leg. Grodno (Grodna) Prov., Smorgon' Distr., 2 km SW Rachuny Vill., forest road, 2 Sept. 2000, Tussilago farfara L., on lower side of leaves, apt., S.V. Buga leg. Vitebsk Prov., Miory Distr., $2 \mathrm{~km}$ SSW Yurkovshchina Vill., ruderal vegetation, 2 July 2007, Tussilago farfara L., on lower side of leaves, apt., S.V. Buga leg.

\section{Uroleucon (Lambersius) erigeronense (Thomas, 1878)}

Specimens examined. Minsk Prov., Smolevichi Distr., near Berezovaya Gora Vill., ruderal vegetation, 21 June 2008, Phalacroloma septentrionale (Fernald et Wiegand) Tzvelev, on stem, apt., S.V. Buga leg. Vitebsk Prov., Lepel' Distr., Lepel', ruderal vegetation, 21 Aug. 2006, Conyza canadensis (L.) Cronq., on stem, apt., N.V. Leshchinskaya leg.

\section{Uroleucon (Uromelan) aeneum (Hille Ris Lambers, 1939)}

Specimens examined. Minsk Prov., Minsk Distr., near Zabolot'e Vill., ruderal vegetation, 26 July 2006, Cirsium arvense (L.) Scop., on stem, apt., N.V. Leshchinskaya leg.

\section{Uroleucon (Uromelan) campanulae}

(Kaltenbach, 1843)

Specimens examined. Minsk Prov., Molodechno Distr., near Udranka Vill., dry meadow, 22 June 2006, Jasione montana L., on stem near inflorescences, apt., N.V. Leshchinskaya leg.

\section{Uroleucon (Uromelan) jaceae \\ (Linnaeus, 1758)}

Specimens examined. Minsk Prov., Minsk Distr., near Roshcha RWS, dry meadow, 21 Oct. 1997, Centaurea jacea L., on apical part of stem, male, S.V. Buga leg. Vitebsk Prov: Gorodok Distr:: Zadrach'e (Zadrachje) Vill., 3 July 1996, Centaurea jacea L., on stem, apt., S.V. Buga leg.; near Makhalovo Vill., 3 June 2000, Centaurea scabiosa L., on stem, apt., S.V. Buga leg.; nearby Zadrach'e (Zadrachje) Vill., 5 Aug. 2007, Centaurea scabiosa L., on apical part of stem, apt., S.V. Buga leg.; Vitebsk Distr., Vitebsk, ruderal vegetation, 29 July 2006, Centaurea jacea L., on stem, N.V. Leshchinskaya leg.

\section{Uroleucon (Uromelan) nigrocampanulae} (Theobald, 1928)

Specimens examined. Vitebsk Prov., Polotsk Distr., 5 km SW Dretun' RWS, pine forest, 2 July 2007, Campanula trachelium L., on apical part of stem, apt., S.V. Buga leg. Published information. Vitebsk Prov., BBR (as Uroleucon trachelii Börn.) (Buga, 1989).

\section{Uroleucon (Uromelan) rapunculoidis (Börner, 1939)}

Specimens examined. Vitebsk Prov., Gorodok Distr., near Rudnia (Rudnya, Rudnja) Vill., 18 June 2008, Campanula patula L., on stem near inflorescences, apt., al., S.V. Buga leg.

\section{Uroleucon (Uromelan) simile \\ (Hille Ris Lambers, 1935)}

Specimens examined. Grodno (Grodna) Prov., Korelichi Distr., near Korelichi, dry meadow, 4 Aug. 2008, Conyza canadensis (L.) Cronq., on stem, apt., N.V. Leshchinskaya leg. 


\section{Uroleucon (Uromelan) solidaginis}

(Fabricius, 1779)

Specimens examined. Brest Prov., $\mathrm{Ka}$ menets Distr., BPF, near Kamenyuki Vill., pine forest, 21 Sept. 2000, Solidago virgaurea L., on apical part of stem, males, ovip. fem., S.V. Buga leg. Minsk Prov., Volozhin Distr., NBR, hamlet Pechishche, dry meadow, 26 July 1987, Solidago virgaurea L., on lower side of leaves, apt., S.V. Buga leg. Vitebsk Prov.: Gorodok Distr.: Sinyaki (Sinjaki) Vill., 3 July 1996, Solidago virgaurea L., apices of stem, apt., S.V. Buga leg.; Zadrach'e (Zadrachje) Vill., 12 July 2000, Solidago virgaurea L., on stem near inflorescences, apt., al., S.V. Buga leg.; Rudnia (Rudnya, Rudnja) Vill., 18 June 2008, Solidago virgaurea L., apices of stem, apt., S.V. Buga leg.; Miory Distr., near Yazno Vill., mixed forest, 22 June 2007, Solidago virgaurea L., apices of stem, apt., N.V. Leshchinskaya leg.

\section{Uroleucon (Uromelan) taraxaci}

(Kaltenbach, 1843)

Specimens examined. Minsk, CBG, ruderal vegetation, 15 June 2006, Taraxacum officinale L. s.l., on lower side of leaves, apt., N.V. Leshchinskaya leg.

\section{Wahlgreniella ossiannilssoni \\ Hille Ris Lambers, 1949}

Specimens examined. Vitebsk Prov.: Gorodok Distr., $0.3 \mathrm{~km}$ ENE Marchenki Vill., pine forest, 13 July 2000, Arctostaphylos uva-ursi (L.) Spreng., on lower side of leaves, apt., V.E. Jarigo leg.; Lepel' Distr., BBR, near hamlet Savskiy Bor, pine forest, 8 July 2004, Arctostaphylos uva-ursi (L.) Spreng., on apical part of stem, apt., al., S.V. Buga leg.

\section{Wahlgreniella vaccinii (Theobald, 1924)}

Specimens examined. Grodno (Grodna) Prov., Smorgon' Distr., 3.3 km SW Rachuny Vill., pine forest, 2 Sept. 2000, Vaccinium
(Rhodococcum) vitis-idaea L., on lower side of leaves, apt., S.V. Buga leg.

\section{DISCUSSION}

Two species earlier reported from Belarus were excluded from the list: Dysaphis flava Shaposhnikov, 1952 and D. crataegi (Kaltenbach, 1843). Dysaphis flava was first mentioned by Buga (2001) with reference to Shaposhnikov (1964); however, re-examination of the cited publication revealed that Shaposhnikov (1964) did not record this species directly in Belarus. The second species was reported by Gorlenko \& Pan'ko (1972) but, in our opinion, this was a case of misidentification because the cited authors did not use appropriate methods to distinguish these species from morphologically similar ones.

Now 156 species and 2 subspecies of Macrosiphini (belonging to 52 genera) are recorded in Belarus. Acyrthosiphon cyparissiae (Koch, 1855), A. euphorbiae Börner, 1949, Diuraphis (Holcaphis) frequens (Walker, 1848), Dysaphis angelicae (Koch, 1854), D. apiifolia petroselini (Börner, 1950), D. lauberti (Börner, 1940), Paramyzus heraclei Börner, 1933, Macrosiphum melampyri Mordvilko, 1919, Uroleucon pulicariae (Hille Ris Lambers, 1939) are recorded in Belarus for the first time. The taxonomic diversity is not so rich in comparison with that in the adjacent countries - Latvia (174 species, 61 genera (Rupais, 1989)) and Poland (317 species, 83 genera (Osiadacz \& Hałaj, 2009)). One of the reasons behind the smaller number of species recorded from Belarus is the absence of the seashore habitats, from which several stenotopic species were recorded in both Latvia and Poland (including in particular Megoura littoralis F.P. Müller, 1972). Territory of Poland (in contrast to Belarus) also includes mountains, forest-steppes and steppes with specific aphid fauna. Nevertheless, comparison with the adjacent countries makes it possible to speculate that some 60-100 additional Macrosiphini species are likely to be found in Belarus. 


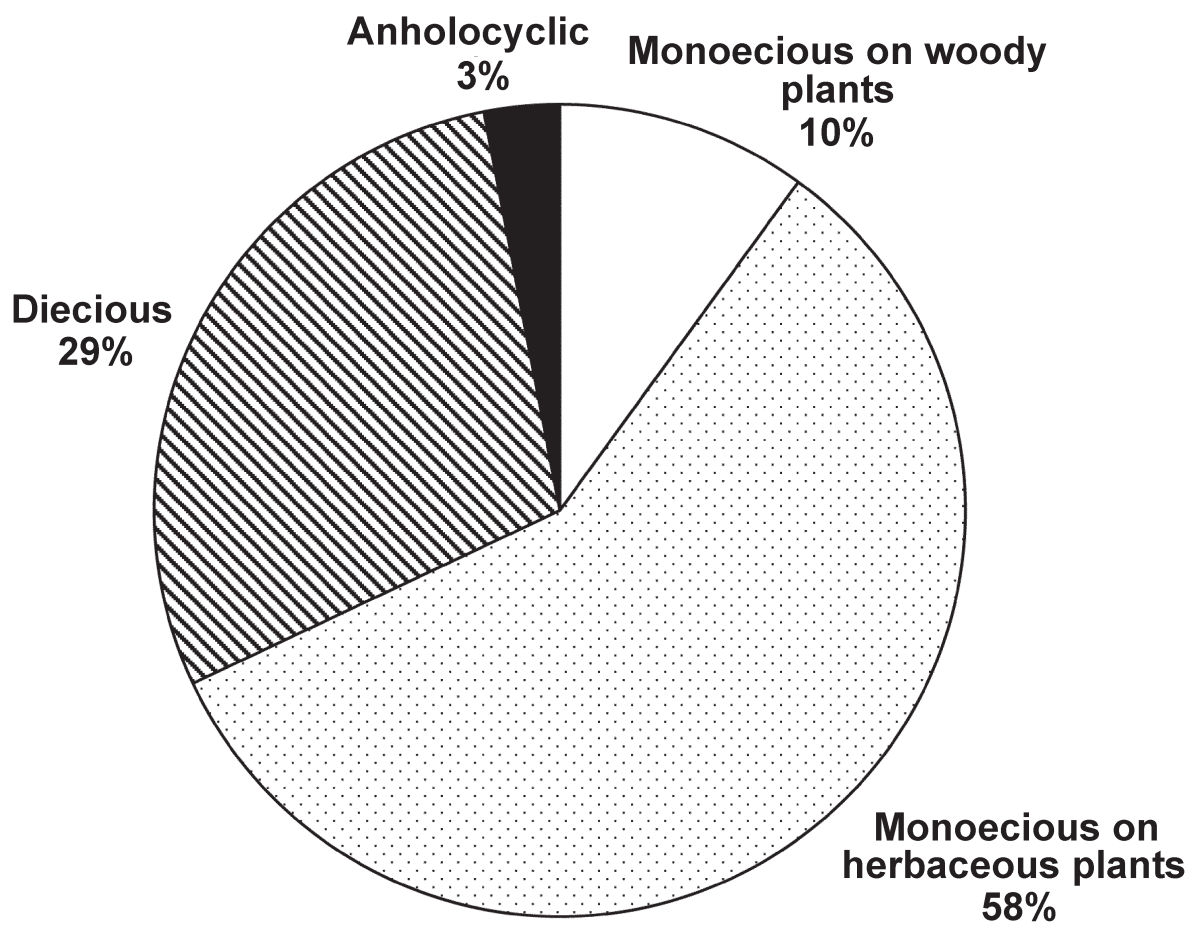

Fig. 3. Relative presence in Belarusian fauna of Macrosiphini species with different types of life cycles.

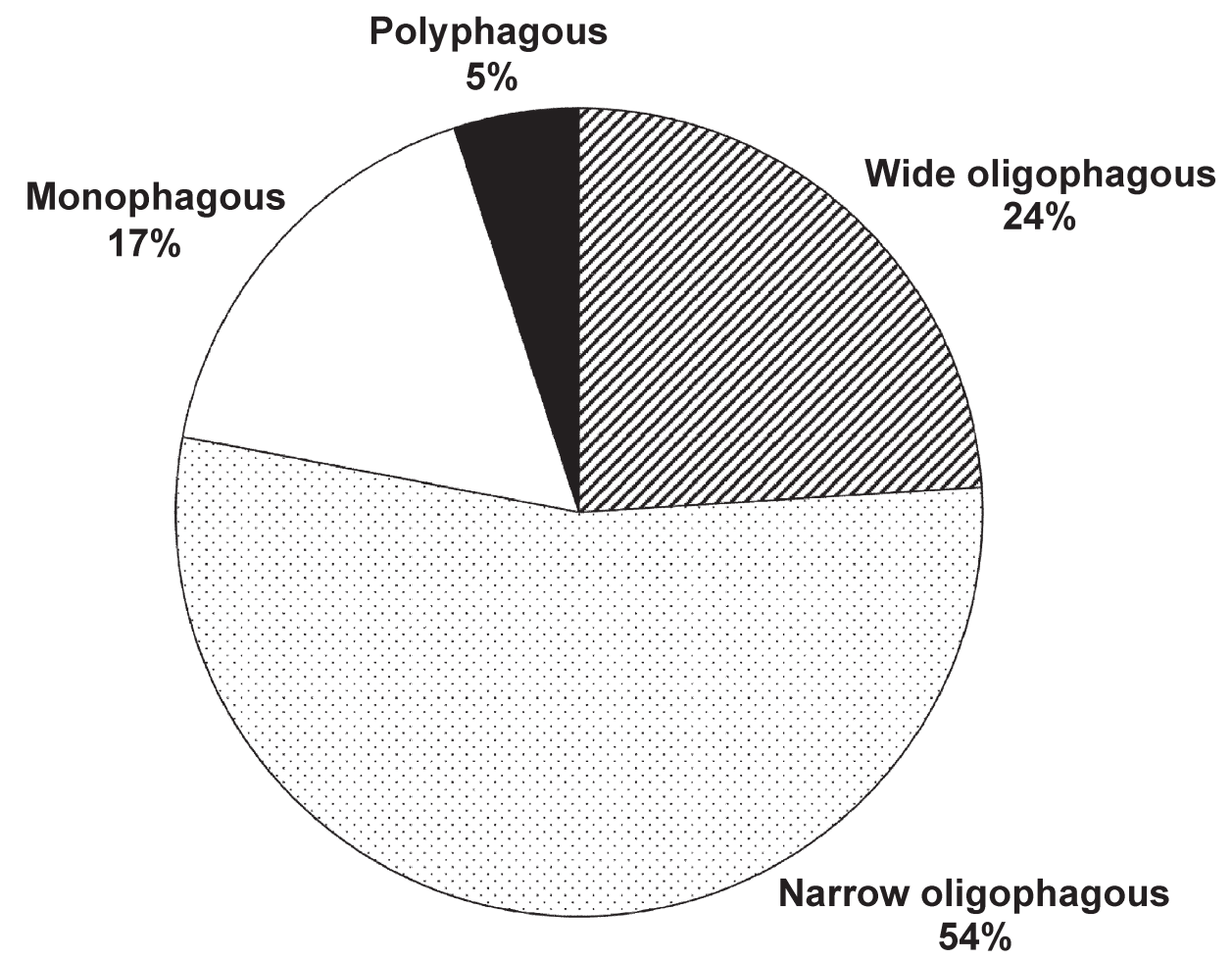

Fig. 4. Relative presence in Belarusian fauna of Macrosiphini species with different types of trophic specialization. 


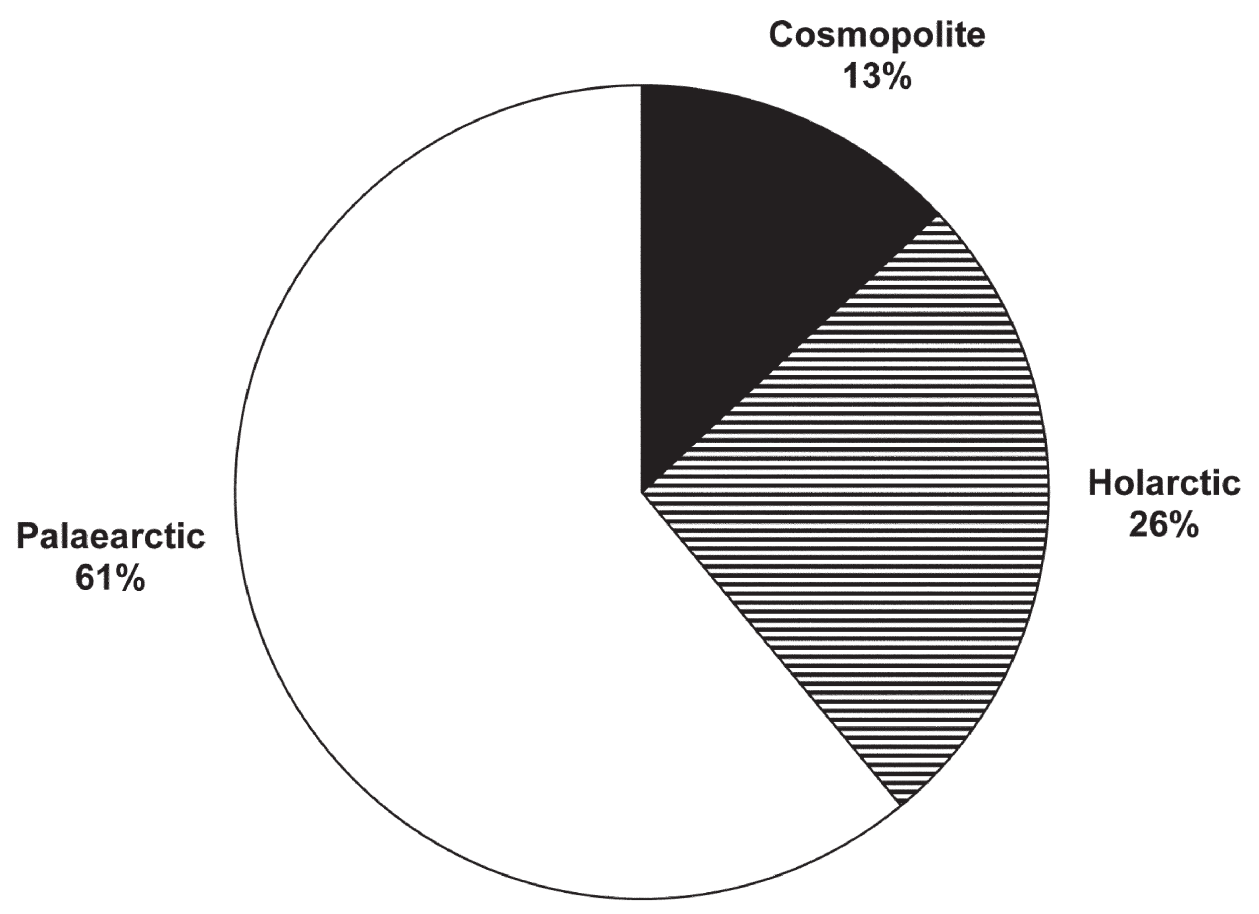

Fig. 5. Relative presence in Belarusian fauna of Macrosiphini species with different types of geographic distribution.

Among the landscape provinces of Belarus (Fig. 2), the fauna of Macrosiphini has been best studied in Western Belarus. Data from Belarus Lakeland (Poozer'e) originate mainly from the Gorodock Highland (Rakauskas \& Buga, 2010). In general, the number of recorded species reflects mostly collecting efforts rather than biogeographical differences between provinces.

In line with the global pattern of host plant use (Shaposhnikov, 1985), the majority of Macrosiphini species recorded in Belarus (Fig. 3) permanently inhabit herbaceous plants (monoecious species) or migrate to them from woody plants (dioecious species). Among the recorded species, 27 feed on a single host plant, 85 on hosts from one genus, 37 on hosts from one family, and only seven species use host plants from more than one family (Fig. 4).

The majority of recorded species have geographical distribution in the Palaearctic or
Holarctic (Fig. 5). At least 16 species (10\% of the local Macrosiphini fauna) expanded to Belarus with introduced plants or by invasion from adjacent areas. The probable origin of these alien species is suggested on the basis of geographical distribution of their host plants prior to human impact. Eleven of these species feed on introduced plants only (Table 1). A number of Macrosiphini species (such as Macrosiphoniella sanborni, Myzus ascalonicus, Myzus persicae, and Neomyzus circumflexum) cannot overwinter out of doors under the condition of Belarus. The introduction history is more or less documented for three species only. J. Holman (1971) discussed the distribution of Impatientinum asiaticum briefly after it had been discovered in Europe and gave arguments for the hypothesis that its recent rapid spreading from Asia took place by means of aeroplanes. Brachycorynella lonicerina (feeding on aboriginal Lonicera xylosteum L.) has 
Table 1. Invasive species in recent Macrosiphini fauna of Belarus.

\begin{tabular}{|c|c|c|}
\hline Aphid species & Native host plant(s) & Probable geographical origin \\
\hline $\begin{array}{l}\text { Acyrthosiphon caraganae } \\
\text { (Cholodkovsky, 1908) }\end{array}$ & Caragana arborescens Lam. & Central Asia (Altai) \\
\hline $\begin{array}{l}\text { Brachycaudus spiraeae } \\
\text { Börner, } 1932\end{array}$ & Spiraea salicifolia $\mathrm{L}$. & Central or Southern Europe \\
\hline $\begin{array}{l}\text { Capitophorus elaeagni } \\
\text { (del Guercio, 1894) }\end{array}$ & Elaeagnus spp., Hippophae rhamnoides L. & Arid zone of Eurasia \\
\hline $\begin{array}{l}\text { Capitiphorus hippophaes } \\
\text { (Walker, 1852) }\end{array}$ & Elaeagnus spp., Hippophae rhamnoides L. & Arid zone of Eurasia \\
\hline $\begin{array}{l}\text { Capitophorus pakansus } \\
\text { Hottes et Frison, } 1931\end{array}$ & Elaeagnus spp., Hippophae rhamnoides L. & Arid zone of Eurasia \\
\hline $\begin{array}{l}\text { Capitophorus similis } \\
\text { van der Goot, } 1915\end{array}$ & Elaeagnus spp., Hippophae rhamnoides L. & Arid zone of Eurasia \\
\hline $\begin{array}{l}\text { Hyadaphis tataricae } \\
\text { (Aizenberg, 1935) }\end{array}$ & Lonicera tatarica $\mathrm{L}$. & $\begin{array}{l}\text { Eastern Europe } \\
\text { (Ural and Volga region) }\end{array}$ \\
\hline $\begin{array}{l}\text { Impatientinum asiaticum asiaticum } \\
\text { Nevsky, } 1929\end{array}$ & Impatiens parviflora $\mathrm{DC}$. & Central Asia \\
\hline $\begin{array}{l}\text { Macrosiphum albifrons } \\
\text { Essig, } 1911\end{array}$ & Lupinus spp. & North America \\
\hline Myzus cerasi (Fabricius, 1775) & Cerasus spp. & Southern Europe \\
\hline Myzus ligustri (Mosley, 1841) & Ligustrum vulgare $\mathrm{L}$. & Central or Southern Europe \\
\hline
\end{tabular}

spread from the Ural and Volga regions during the 20th century (Vereshchagin et al., 1985). Acyrthosiphon caraganae (feeding on Caragana arborescens Lam. introduced from Central Asia) probably originated from the Altai (Mordvilko, 1914).

\section{ACKNOWLEDGEMENTS}

We are grateful to Yu.V. Safonova, I.P. Moskvina, V.E. Jarigo, A.A. Mityanin, A.A. Ponomarev, D.L. Petrov, N.V. Leshchinskaya, R. Rakauskas, L.A. Serbina, N.V. Voronova, O.L. Nesterova for providing aphid materials, to T.A. Sautkina (Belarusian State University, Minsk) for help with identification of host plants and to M.V. Kozlov (University of Turku, Finland) for critical revision of the earlier version of the manuscript. The collection of Zoological Institute of the Russian Academy of Sciences was financially supported by the Ministry of Education and Science of the Russian Federation (project no. 16.518.11.7070).

\section{REFERENCES}

Bezdenko T.T. 1958. Zashchita plodovogo sada ot vrediteley [Fruit orchard protection from pests]. Minsk: Zvyazda. 122 p. (In Russian).

Blackman R.L. \& Eastop V.F. 2000. Aphids on the World's Crops, An Identification and Information Guide, 2nd Edition. Chichester: John Wiley \& Sons, Ltd. $476 \mathrm{p}$

Blackman R.L. \& Eastop V.F. 2011. Additions and amendments to "Aphids on the World's Plants". Zootaxa, 2774, 57-68.

Buga S.V. 1989. Order Homoptera. In: Sokolov V.E. (Ed.) Flora i fauna zapovednikos SSSR: Nasekomye Berezinskogo zapovednika [Flora and Fauna of the USSR reserves: Insects of Berezina reserve]: 8-14 pp. Moscow: VINITI. (In Russian). 
Buga S.V. 1995. Dendrocolous aphids (Homoptera: Aphidoidea) of Naliboki reserve. Newsletters of Belarusian University. Ser. 2, 1: 40-43. (In Russian).

Buga S.V. 1997. Dendrocolous aphids (Homoptera: Aphidoidea) of non-park green stands in Minsk. Neresletters of Belarusian University. Ser. 2, 3: 36-39. (In Russian).

Buga S.V. 2001. Dendrofil'nye tli Belorusi [Dendrocolous aphids of Belarus]. Minsk: Belarusian University Publishers. 98 p. (In Russian).

Buga S.V. \& Gorlenka S.U. 1987. Character of dendrocolous aphid complex organization in urban green stands. Vesti Akademii nauk BSSR, Seriya Biologicheskikh nauk [The Belarusian SSR Academy of Sciences Newsletters. Ser. Biological Sciences], 2: 13-17. (In Belarusian).

Buga S.V., Leshchinskaya N.V. \& Stekolshchikov A.V. 2008. Addition to the aphid (Homoptera: Aphidinea) fauna of Belaweza Primeval Forest. Newesletters of Belarusian University. Ser. 2, 2: 57-61. (In Russian).

Buga S.V. \& Stekolshchikov A.V. 2009. Aphids as pests of fruit- and berry-producing plants in Byelorussia. Redia, 92: 239-242.

Eastop V.F. \& Blackman R.L. 2005. Some new synonyms in Aphididae (Hemiptera: Sternorrhyncha). Zootaxa, 1089: 1-36.

Fiedorowiczówna Z. 1930. Zoocecidia roślin zebranych w powiece Dziśnieńskim i Brasławskim na Ziemi Wileińskiej. Prace Towarzystwa Przyjaciol Nauk w Wilnie. Wydziat nauk matematycznych i przyrodnych, 6: 25-59. (In Polish).

Gorlenko S.V. \& Pan'ko N.A. 1967. Vrediteli $i$ bolezni introdutsirovannykh rasteniy [Pests and diseases of introduced plants]. Minsk: Nauka i tekhnika. 136 pp. (In Russian).

Gorlenko S.V. \& Pan'ko N.A. 1972. Formirovanie mikroflory i entomofauny gorodskih zelenykh nasazdeniy [Genesis of microflora and entomofauna in urban green stands]. Minsk: Nauka i tekhnika. 168 pp. (In Russian).

Holman J. 1971. Taxonomy and ecology of Impatientinum asiaticum Nevsky, an aphid species recently introduced to Europe (Homoptera, Aphididae). Acta Entomologica Bohemoslovaca, 68: 153-166.

Holman J. 2009. Host Plant Catalog of Aphids: Paleartic Region. Netherlands: Springer. $1140 \mathrm{p}$.
Kharchanka N.N. 1968. Some biological characteristics of powder plum aphid in Belarus. Vestsy Akademii navuk BSSR, Seriya sel'skagaspadartsykh navuk [Newsletters of the Belarusian SSR Academy of Sciences, Series of the Agricultural Sciences], 3: 7173. (In Belarusian).

Losinskaya N.L. 1960. The main representatives of tree and shrub pest entomofauna at the Central Botany Gardens of the BSSR Academy of Sciences. Central'nyy Botanicheskiy Sad Akademii Nauk BSSR: Sbornik nauchnykh rabot [The Central Botany Gardens of the Belarusian SSR Academy of Science: Miscellaneous of scientific works], 1: 114-121. (In Russian).

Martsinkevich G.I. \& Pirozhnik I.I. 2007. Physical-geographical subdivision of Belarus in decimal system. In: Ostaszewska K., Szumacher I., Kulczyk S. \& Malinowska E. (Eds.) Znaczenie badan krajobrazowych dla zrownowazonego rozwoju [The importance of landscape research for sustainable development]: 361-370. Warszawa: Wydawnictwa Uniwersytetu Warszawskiego.

Mordvilko A.K. 1914. Fauna Rossii i sopredelnykh stran [Fauna of Russia and Adjacent Countries. Insecta Hemiptera], 1 (1). Petrograd: Imperatorskaya Akademiya Nauk. 236 +9 p. (In Russian).

Mordvilko A.K. 1930. The preliminary report on Belarusian aphid (Aphidae) fauna investigations in Belarus in summer 1929. Materials for Belarus flora and fauna investigation, 5, 161-164. (In Belarusian).

Mordvilko A.K. 1932. I. Pests of field, vegetable, industrial and pharmaceutical crops. D Suborder Aphidodea. Aphids. In: Stackelberg A.A. (Ed.) Spisok vrednykh nasekomykh SSSR $i$ sopredel'nykh stran. Chast' I. Vrediteli selskogo khozyaystva. [List of insect pests of the USSR and adjacent countries. Part I. Agricultural pests.] Bulletin of plant protection, I ser.: Entomology, 5: 48-63. (In Russian).

Mormyljova V.F. 1981. Aphids of grain cereals in Belarus and measures of its control. In Aukshtikal'nene A.M., Zayanchkauskas A.P., Semyanov V.P. \& Yakimavichus A.B. (Eds.) Noveyshie dostizheniya sel'skokhozyaystvennoy entomologii (Trudy vos'mogo s'ezda Vsevoyuznogo entomologicheskogo obshchestva, Vilnus, 9-13 oktyabrya 1979) [Newest achievements of agricultural Entomology (Proceedings of the 8th congress of 
the All-union entomological society, Vilnius, October, 9-13, 1979)]: 119-122. Vilnius. (In Russian).

Osiadacz B. \& Hałaj R. 2009. The aphids (Hemiptera: Sternorrhyncha: Aphidinea) of Poland. A Distributional Checklist. Polish Entomological Monographs, 6: 1-96.

Pan'ko N.A. 1969. On pest fauna of urban green stands in the South of Belarus. In: Smolsky N.V. (Ed.) Introduktsiya rasteniy $i$ okhrana prirody [Plant introduction and environmental protection]: 204-214. Minsk: Nauka i tekhnika. (In Russian).

Parfenov V.I. (Ed.) 1999. Opredelitel' vysshikh rasteniy Belarusi [Higher plants of Belarus: keybook]. Minsk: Design PRO. 472 p. (In Russian).

Perepeczko-Baumanowa J. 1934. Zoocecidia zebrane w Oszmianie i najbliższych okolicach. Prace Towarzystwa Przyjaciol Nauk w Wilnie. Wydziat nauk matematycznych i przyrodnych, 9: 239-261.

Rakauskas R. \& Buga S. 2010. Contribution to the knowledge of the aphid (Hemiptera, Sternorrhyncha: Aphidoidea) fauna of the Gorodok Highland, Belarus. Acta Zoologica Lituanica, 20 (4): 205-224.

Remaudière G. \& Remaudière M. 1997. Catalogue des Aphididae du monde-Catalogue of the word's Aphididae (Homoptera, Aphidoidea). Paris: INRA editions. 473 p.

Rupais A.A. 1989. Tli (Aphidodea) Latvii (Aphidodea) [The aphids (Aphidodea) of Latvia]. Riga: Zinatne. 331 p. (In Russian).

Shaposhnikov G.Ch. 1964. Suborder Aphidinea-Aphids. In: Bei-Bienko G.Ya. (Ed.)
Opredelitel' nasekomykh Evropeyskoy chasti $S S S R$ [A Key to the insects of the European part of the USSR], 1: 489-616. Moscow, Leningrad: Nauka. (In Russian).

Shaposhnikov G.Ch. 1985. The main features of the evolution of aphids. In: Evolution and biosystematics of aphids. Proceedings of the International Aphidological Symposium at Jablonna, Poland, April 1981: 19-99. Wroclaw: Ossolineum.

Stekolshchikov A.V. \& Buga S.V. 2006. Notes on Trichosiphonaphis (Xenomysus) cortices Aizenberg, 1935 and T. (X.) foliotus Shaposhnikov in Juchnevitch, 1968, nomen nudum (Homoptera: Aphididae). Zoosystematica Rossica, 15 (1): 63-68.

Stekolshchikov A.V., Buga S.V. \& Leshchinskaya N.V. 2008. Addition to the aphid fauna of Byelorussia (Homoptera: Aphidoidea) with detailed description of sexuales of Semiaphis anthrisci (Kaltenbach, 1843). Zoosystematica Rossica, 17 (1): 61-66.

Stekolshchikov A.V., Leshchinskaya N.V., Buga S.V. \& Voronova N.V. 2010. Recent additions to the aphid (Homoptera: Aphidoidea) fauna of Byelorussia. Zoosystematica Rossica, 19 (1): 35-38.

Vereshchagin B.V., Andreev A.V. \& Vereshchagina A.B. 1985. Tli Moldavii [Aphids of Moldavia]. Chisinau: Stiinta. 158 p. (In Russian).

Zhukova M.I. 1986. Potato leafroll virus and some of the reasons for its spread in Belarus. Zashchita Rasteniy, 11: 45-51. (In Russian).

Received December 27, 2011 / Accepted May 20, 2012 